\title{
Entanglement entropy of Wilson surfaces from bubbling geometries in M-theory
}

Simon A. Gentle, Michael Gutperle and Chrysostomos Marasinou

Department of Physics and Astronomy, University of California, Los Angeles, CA 90095, U.S.A.

E-mail: sgentle@physics.ucla.edu, gutperle@physics.ucla.edu, cmarasinou@physics.ucla.edu

ABSTRACT: We consider solutions of eleven-dimensional supergravity constructed in $[1,2]$ that are half-BPS, locally asymptotic to $A d S_{7} \times S^{4}$ and are the holographic dual of heavy Wilson surfaces in the six-dimensional $(2,0)$ theory. Using these bubbling solutions we calculate the holographic entanglement entropy for a spherical entangling surface in the presence of a planar Wilson surface. In addition, we calculate the holographic stress tensor and, by evaluating the on-shell supergravity action, the expectation value of the Wilson surface operator.

KEYwORDs: AdS-CFT Correspondence, M-Theory, Gauge-gravity correspondence

ARXiv EPRINT: 1506.00052 


\section{Contents}

1 Introduction $\quad 2$

1.1 Summary of results 3

1.2 Structure of the paper 4

2 Review of bubbling M-theory solutions 4

2.1 Asymptotic behaviour and regularization 7

3 Holographic entanglement entropy $\quad 9$

$\begin{array}{lll}3.1 & \text { Minimal surface geometry } & 10\end{array}$

$\begin{array}{ll}3.2 & \text { Evaluating the area integral }\end{array}$

$\begin{array}{lll}3.3 & \text { Physical interpretation } & 12\end{array}$

4 Holographic stress tensor $\quad 13$

5 Expectation value of the Wilson surface operator $\quad \mathbf{1 5}$

$\begin{array}{ll}5.1 \text { Action as a total derivative } & 15\end{array}$

$\begin{array}{ll}5.2 \text { Gibbons-Hawking term } & 17\end{array}$

$\begin{array}{lll}5.2 .1 & \text { Real line contribution } & 18\end{array}$

$\begin{array}{lll}5.2 .2 & \text { Large } r \text { contribution } & 18\end{array}$

$\begin{array}{lll}5.3 & \text { Bulk supergravity action } & 19\end{array}$

$\begin{array}{lll}\text { 5.3.1 Large } r \text { contribution } & 20\end{array}$

5.3.2 Real line contribution 20

5.4 Final result 21

6 Discussion $\quad 22$

A Contributions to the entanglement entropy 23

A.1 $J_{1}$

A.2 $J_{2} \quad 24$

$\begin{array}{ll}\text { B Calculation of the holographic stress tensor } & 27\end{array}$

$\begin{array}{ll}\text { C Four-form field strength } & 29\end{array}$

D Calculation of the real line contribution to the on-shell action 30 


\section{Introduction}

The celebrated proposal of Ryu and Takyanagi $[3,4]$ provides a method to calculate entanglement entropies for field theories that have holographic duals. Originally the proposal was used to calculate the entanglement entropy for theories in their vacuum state, but was quickly generalized to include more general settings, such as finite temperature and timedependent states (see e.g. [5] for a review). For spherical entangling surfaces it was observed by Casini, Huerta and Myers [6] that the holographic entanglement entropy can be mapped to the thermal entropy of a hyperbolic black hole. In the field theory, the corresponding entanglement entropy is mapped to the thermal entropy on a hyperbolic space.

Another generalization concerns entanglement entropy in the presence of extended defects, such as Wilson lines. In the probe approximation these defects are described by strings or branes in the AdS space and the backreaction of the stress energy of the branes on the bulk geometry is neglected. For example, the holographic description of a Wilson loop in $\mathrm{SU}(N) \mathcal{N}=4 \mathrm{SYM}$ in the fundamental representation is given by a fundamental string in $A d S_{5} \times S^{5}[7,8]$, whereas higher dimensional representations can be described by D3- (D5-) branes with $A d S_{2} \times S^{2}\left(S^{4}\right)$ worldvolume in $A d S_{5} \times S^{5}[9,10]$. See [11-13] for a discussion of entanglement entropy in the presence of probe brane defects. When the dimension of the representation increases and becomes of order $N^{2}$, the backreaction cannot be neglected and the probe is replaced by a new bubbling geometry with flux. The bubbling holographic solutions corresponding to half-BPS Wilson loops in $\mathcal{N}=4 \mathrm{SYM}$ were found in [14-16].

In [17], Lewkowycz and Maldacena applied the Casini, Huerta and Myers mapping to the calculation of the entanglement entropy in the presence of Wilson loops in $\mathcal{N}=4$ SYM theory and ABJM theories [18]. They showed that the entanglement entropy can be calculated from the expectation value of the Wilson loop operator as well as the one point function of the stress tensor in the presence of the Wilson loop. For BPS Wilson loops these quantities can be evaluated using localization and reduced to matrix models [19, 20]. In [21] by two of the present authors, the holographic entanglement entropy was calculated using the bubbling solution dual to half-BPS Wilson loops. It was shown that the result agrees with [17] when the exact map between matrix model quantities and the supergravity solution, found in $[22,23]$, is applied.

The goal of the present paper is to generalize the holographic calculation of the entanglement entropy to the case of six-dimensional $(2,0)$ theory with half-BPS Wilson surfaces present. The $(2,0)$ theory can be defined either by a low-energy limit of type IIB string theory on an $A_{N-1}$ singularity [24] or by a decoupling limit of $N$ coincident M5 branes [25]. While there exists no simple Lagrangian description of this theory due to the presence of tensor fields $B^{+}$with self-dual field strength, the holographic dual [26] is given by M-theory on $A d S_{7} \times S^{4}$. In analogy with the Wilson loop one expects that the six-dimensional theory has extended Wilson surface operators [27] of the form

$$
W_{\Gamma} \sim \operatorname{tr} \exp \left(\int_{\Gamma} B^{+}\right)
$$




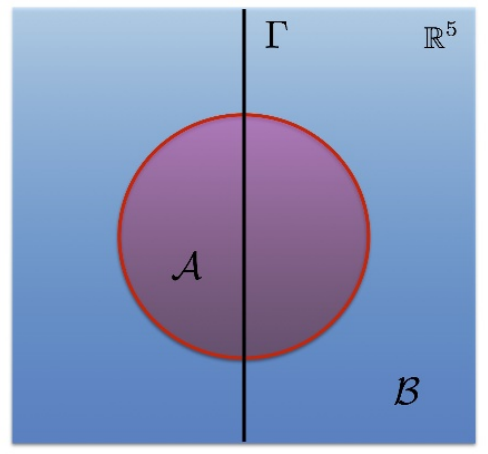

Figure 1. The spherical entangling surface $\partial \mathcal{A}$ is the boundary of a region $\mathcal{A}$ on a constant time slice of the $(2,0)$ theory on $\mathbb{R}^{6}$. The Wilson surface $\Gamma$ intersects this surface twice.

In the probe approximation the Wilson surface operators can be described by embedding M2-branes [28, 29] or M5-branes [30-32] on various submanifolds inside the $A d S_{7} \times S^{4}$. It is an interesting open question whether the expectation value of Wilson surface operators can be calculated by localization in the $(2,0)$ theory.

In $[1,2]$, bubbling solutions corresponding to half-BPS Wilson surfaces were found (see [33] for earlier work in this direction). In the present paper we will use these solutions of eleven-dimensional supergravity to calculate the entanglement entropy as well as other holographic observables. The bubbling solutions feature an $A d S_{3} \times S^{3} \times S^{3}$ fibration over a two-dimensional Riemann surface $\Sigma$ with boundary. The solutions are locally asymptotic to $A d S_{7} \times S^{4}$ and the six-dimensional asymptotic metric on the $A d S_{7}$ boundary is naturally $A d S_{3} \times S^{3}$. It is convenient to describe the Wilson surface on this space by imposing boundary conditions at the boundary of $A d S_{3}$ and choosing Poincaré coordinates for the $A d S_{3}$ factor describes a planar Wilson surface. However, as discussed in section 3.3, this metric on $A d S_{3} \times S^{3}$ can be related to the more familiar flat metric on $\mathbb{R}^{6}$ by a conformal transformation. It is easier to visualize the geometry of our setup on $\mathbb{R}^{6}$ : the entangling surface at constant time is a four-sphere of radius $R$ and the Wilson surface is a line (also filling out the time direction) that intersects the four-sphere at two points, as illustrated in figure 1.

\subsection{Summary of results}

For the convenience of the reader we will present our main results here and put them into context. The change of the entanglement entropy due to the presence of the Wilson surface is given by

$$
\Delta S_{\mathcal{A}}=\frac{4 N^{3}}{3}\left[\frac{16+3 m_{2}^{2}-8 m_{3}}{320}-\frac{1}{16} \sum_{i<j}(-1)^{i+j}\left|\xi_{i}-\xi_{j}\right|^{3}-\frac{1}{2}\right] \log \left(\frac{2 R}{\eta}\right)
$$

We also calculate two other holographic observables. The stress tensor in the presence of the Wilson surface for the $A d S_{3} \times S^{3}$ boundary coordinates is given by

$$
\Delta\left\langle T_{i j}\right\rangle d x^{i} d x^{j}=\frac{N^{3}}{160 \pi^{3}}\left(16+3 m_{2}^{2}-8 m_{3}\right)\left(d s_{A d S_{3}}^{2}-d s_{S^{3}}^{2}\right)
$$


Finally we calculate the expectation value of the Wilson surface operator by evaluating the regularized on-shell supergravity action:

$$
\log \left\langle W_{\Gamma}\right\rangle=-\frac{N^{3}}{192 \pi} \operatorname{Vol}\left(A d S_{3}\right)(\mathcal{F}+64)
$$

The $m_{2,3}$ are quantities that depend on the parameters $\xi_{i}$ of a general bubbling solution. The cut-off $\eta$ is the distance from the Wilson surface, as discussed in more detail in section 3.3. Also, $\mathcal{F}$ is a finite one-dimensional integral that is defined in section 5.3.2.

\subsection{Structure of the paper}

The structure of the paper is as follows. In section 2 we review the bubbling half-BPS solutions of M-theory originally obtained in $[1,2]$ and work out the behavior of the solution near the asymptotic boundary. In particular, we determine the Fefferman-Graham map for an asymptotic $A d S_{3} \times S^{3}$ boundary metric. In section 3 we calculate the entanglement entropy for a spherical entangling surface following the Ryu-Takayanagi prescription for the bubbling solution. In section 4 we use the methods of Kaluza-Klein holography and holographic renormalization to calculate the one point function of the stress tensor for the bubbling solution. In section 5 we evaluate the on-shell action of eleven-dimensional supergravity to determine the expectation value of the Wilson surface. We show that the bulk part of the action is given by a total derivative and evaluate the integral as well as the Gibbons-Hawking term. In section 6 we provide a brief discussion of our results as well as possible avenues for future research. In the interest of readability we present technical matters and details of calculations in several appendices.

\section{Review of bubbling M-theory solutions}

In this section we will review the construction of half-BPS M-theory solutions found in [1] that are locally asymptotic to $A d S_{7} \times S^{4}$. These solutions generalize the construction of Janus solutions $[34,35]$ in type IIB to M-theory. They correspond to the holographic description of Wilson surface defects in the six-dimensional $(2,0)$ theory, where the Wilson surface is 'heavy' and the backreaction on the geometry is taken into account.

One demands that these solutions preserve an $\operatorname{OSp}\left(4^{*} \mid 2\right) \oplus \operatorname{OSp}\left(4^{*} \mid 2\right)$ sub-superalgebra of the $\operatorname{OSp}\left(8^{*} \mid 4\right)$ superalgebra of the $A d S_{7} \times S^{4}$ vacuum. This form of the preserved superalgebra is uniquely determined by demanding that the solution has sixteen unbroken supersymmetries and preserves $s o(2,2 \mid \mathbb{R})$ associated with conformal symmetry on the worldvolume of the Wilson surface, so $(4 \mid \mathbb{R})$ corresponding to rotational symmetry in the space transverse to the Wilson surface and an unbroken $s o(4 \mid \mathbb{R})$ R-symmetry [36]. Note that a generalization was recently analyzed in [37] in which the preserved superalgebra is $D(2 \mid 1, \gamma) \oplus D(2 \mid 1, \gamma)$, but we will not discuss this case here.

It follows from these superalgebra considerations that the bubbling BPS solution has an $s o(2,2 \mid \mathbb{R}) \oplus s o(4 \mid \mathbb{R}) \oplus s o(4 \mid \mathbb{R})$ algebra of isometries. Furthermore, the solution preserves sixteen of the thirty-two supersymmetries. The BPS equations were solved in [1] and the 
global regular solutions were found in [2]. The ansatz for the eleven-dimensional metric is given by an $A d S_{3} \times S^{3} \times S^{3}$ fibration over a Riemann surface $\Sigma$ with boundary:

$$
d s^{2}=f_{1}^{2} d s_{A d S_{3}}^{2}+f_{2}^{2} d s_{S^{3}}^{2}+f_{3}^{2} d s_{\tilde{S}^{3}}^{2}+4 \rho^{2}|d v|^{2}
$$

where we denote the complex coordinate of the two-dimensional Riemann surface by $v$. In addition, $d s_{S^{3}}^{2}$ and $d s_{\tilde{S}^{3}}^{2}$ are the metrics on the unit-radius three-spheres and the metric on the unit-radius Euclidean $A d S_{3}$ in Poincaré half-plane coordinates is given by

$$
d s_{A d S_{3}}^{2}=\frac{d z^{2}+d t^{2}+d l^{2}}{z^{2}}
$$

The Wilson surface on the boundary $A d S_{3} \times S^{3}$ fills the $t, l$ directions and is located at $z=0$.

These solutions are parametrized by a harmonic function $h$ and a complex function $G(v, \bar{v})$ that satisfies a first order differential equation:

$$
\partial_{v} G=\frac{1}{2}(G+\bar{G}) \partial_{v} \ln h
$$

It is useful to introduce the combinations ${ }^{1}$

$$
W_{+}=|G-\bar{G}|+2|G|^{2}, \quad W_{-}=|G-\bar{G}|-2|G|^{2}
$$

in terms of which the metric functions in (2.1) are given by

$$
\begin{aligned}
f_{1}^{6} & =4 h^{2}\left(1-|G|^{2}\right) \frac{W_{+}}{W_{-}^{2}}, & f_{2}^{6} & =4 h^{2}\left(1-|G|^{2}\right) \frac{W_{-}}{W_{+}^{2}} \\
f_{3}^{6} & =\frac{h^{2} W_{+} W_{-}}{16\left(1-|G|^{2}\right)^{2}}, & \rho^{6} & =\frac{\left(\partial_{v} h \partial_{\bar{v}} h\right)^{3}}{16 h^{4}}\left(1-|G|^{2}\right) W_{+} W_{-}
\end{aligned}
$$

It was shown in [2] that for a solution to be regular the functions $h$ and $G$ must satisfy the following conditions on the Riemann surface $\Sigma$ and its boundary:

$$
\begin{aligned}
& h=0, \quad G=0,+i, \quad v \in \partial \Sigma \\
& h>0,|G|^{2}<1 \quad v \in \Sigma
\end{aligned}
$$

First we consider the simplest example: the $A d S_{7} \times S^{4}$ vacuum solution. This can be obtained by choosing $\Sigma$ to be the half strip $\Sigma=\{v=p+i q / 2, p>0, q \in[0, \pi]\}$ with

$$
h=-i L^{3}(\cosh (2 v)-\cosh (2 \bar{v})), \quad G=-i \frac{\sinh (v-\bar{v})}{\sinh 2 \bar{v}}
$$

which produces

$$
f_{1}=2 L \cosh p, \quad f_{2}=2 L \sinh p, \quad f_{3}=L \sin q, \quad \rho=L
$$

Hence the $A d S_{7} \times S^{4}$ metric is given by

$$
d s^{2}=4 L^{2}\left(d p^{2}+\cosh ^{2} p d s_{A d S_{3}}^{2}+\sinh ^{2} p d s_{S^{3}}^{2}\right)+L^{2}\left(d q^{2}+\sin ^{2} q d s_{\tilde{S}^{3}}^{2}\right)
$$




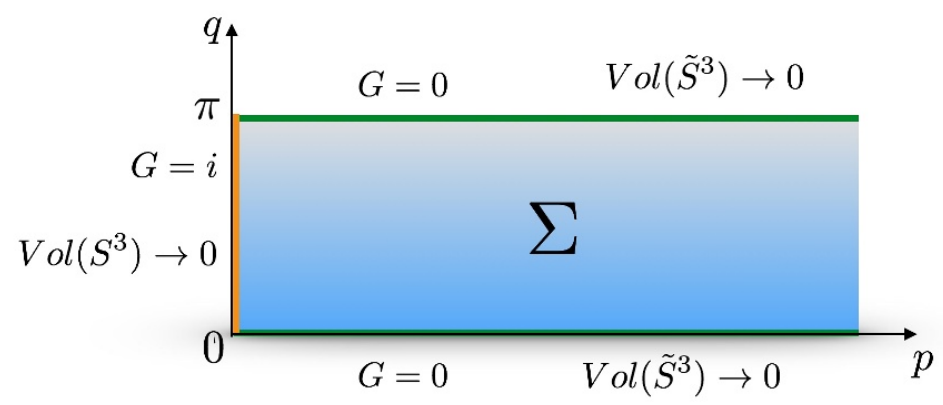

Figure 2. $A d S_{7} \times S^{4}$ parameterized on the half strip.

This geometry is represented in figure 2. More general bubbling solutions can be constructed once we realize that the $A d S_{7} \times S^{4}$ solution can be mapped from the half strip to the upper half-plane via

$$
w=\cosh (2 v)
$$

The functions $h$ and $G$ then take the form

$$
h=-i L^{3}(w-\bar{w}), \quad G=\frac{i}{2}\left(\frac{w+1}{\sqrt{(w+1)(\bar{w}+1)}}-\frac{w-1}{\sqrt{(w-1)(\bar{w}-1)}}\right)
$$

Note that the boundary of $\Sigma$ is now located at the real line and that on the real line the function $G=+i$ when $\operatorname{Re} w \in[-1,1]$ and $G=0$ when $\operatorname{Re} w>1$ or $\operatorname{Re} w<-1$.

A general bubbling solution is constructed by choosing a simple form for $h$ and the following linear superposition for $G$ :

$$
h=-i L^{3}(w-\bar{w}), \quad G=\sum_{i=1}^{2 n}(-1)^{i} g\left(\xi_{i}\right), \quad g(\xi) \equiv-\frac{i}{2} \frac{w-\xi}{\sqrt{(w-\xi)(\bar{w}-\xi)}}
$$

where $w$ is now a general coordinate on the upper half-plane. The solution is completely characterized by the choice of $2 n$ real numbers $\xi_{i}$ with $i=1,2, \ldots, 2 n$ that are ordered

$$
-\infty=\xi_{0}<\xi_{1}<\xi_{2}<\cdots<\xi_{2 n}<\xi_{2 n+1}=+\infty
$$

We have introduced $\xi_{0}$ and $\xi_{2 n+1}$ to simplify the expression for the boundary condition that the function $G$ satisfies on the real line:

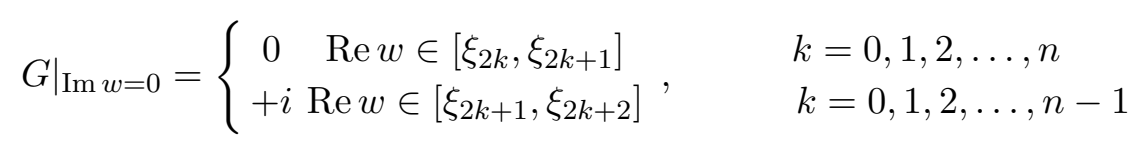

A general bubbling solution is characterized by the appearance of new nontrivial fourcycles (see figure 3 ). The $n$ four-cycles $\tilde{\mathcal{C}}_{4}^{(i)}$ are constructed by connecting two boundary points on different intervals where the volume of the three-sphere $\tilde{S}^{3}$ shrinks to zero. This

\footnotetext{
${ }^{1}$ Note that there is a typo in eq. (2.5) of [2]: it should read $W^{2}=-4|G|^{2}-(G-\bar{G})^{2}$.
} 


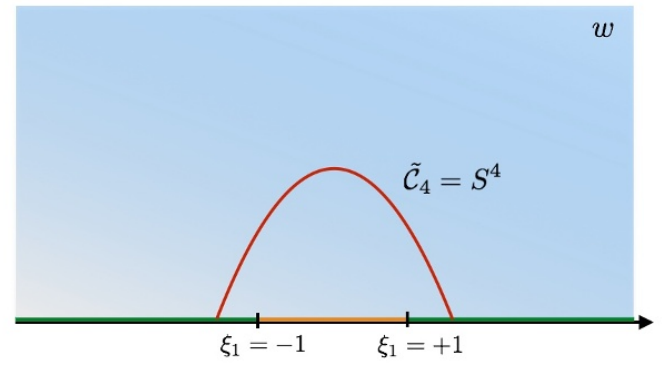

(a)

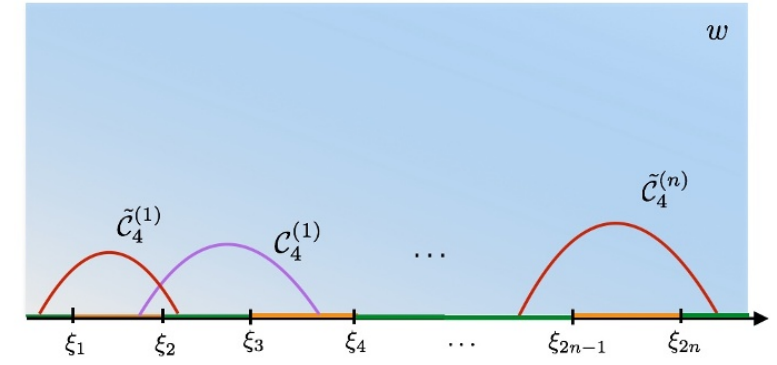

(b)

Figure 3. (a) $A d S_{7} \times S^{4}$ on the upper half-plane. (b) General bubbling solution with $n$ four-cycles $\tilde{\mathcal{C}}_{4}^{(i)}, i=1, \ldots, n$ and $n-1$ four-cycles $\mathcal{C}_{4}^{(i)}, i=1, \ldots, n-1$.

generalizes the construction of the four-sphere in the $A d S_{7} \times S^{4}$ vacuum solution. In addition, the geometry also has $n-1$ four-cycles $\mathcal{C}_{4}^{(i)}$ that are constructed by connecting points on different intervals where the three-sphere $S^{3}$ shrinks to zero size. In the bubbling solution these cycles carry nontrivial four-form flux and are the remnants of M5-branes wrapping $A d S_{3} \times S^{3}$ and $A d S_{3} \times \tilde{S}^{3}$, respectively.

\subsection{Asymptotic behaviour and regularization}

In this section we study the asymptotic behaviour of a general bubbling solution. We will see later that the area integral and the action integral both diverge, so we need to regulate the integrals and map the regulator to the Fefferman-Graham (FG) UV cut-off.

It is convenient to choose the following coordinates on $\Sigma: w=r e^{i \theta}$. The boundary of $A d S_{7} \times S^{4}$ is located at $r \rightarrow \infty$. The expressions for $G$ and $\bar{G}$ given in (2.12) can be expanded at large $r$ in terms of the generating function of the Legendre polynomials

$$
\frac{1}{\sqrt{1-2 x t+t^{2}}}=\sum_{k=0}^{\infty} P_{k}(x) t^{k}
$$

with the result

$$
G=\frac{i}{2} \sum_{k=1}^{\infty} \frac{a_{k}(\theta) m_{k}}{r^{k}}, \quad \bar{G}=-\frac{i}{2} \sum_{k=1}^{\infty} \frac{\bar{a}_{k}(\theta) m_{k}}{r^{k}}
$$

where the dependence on the angular coordinate $\theta$ is given by

$$
\begin{aligned}
& a_{k}(\theta) \equiv P_{k-1}(\cos \theta)-e^{i \theta} P_{k}(\cos \theta) \\
& \bar{a}_{k}(\theta) \equiv P_{k-1}(\cos \theta)-e^{-i \theta} P_{k}(\cos \theta)
\end{aligned}
$$

The moments $m_{k}$ are defined via

$$
m_{k} \equiv \sum_{i=1}^{2 n}(-1)^{i} \xi_{i}^{k}
$$


To ensure that a general bubbling solution is asymptotic to $A d S_{7} \times S^{4}$ with radii

$$
R_{S^{4}}=\frac{R_{A d S_{7}}}{2}=L
$$

we must identify $m_{1} \equiv 2$. This provides a constraint on the $\xi_{i}$. Also, for the $A d S_{7} \times S^{4}$ solution $(n=1)$ we note here that all even moments vanish and all odd moments equal 2 .

The metric functions take the following forms as power series in large $r$ :

$$
\begin{aligned}
\frac{f_{1}^{2}}{L^{2}} & =2 r+\frac{4-m_{2} \cos \theta}{2}+\frac{3\left(8+m_{2}^{2}-2 m_{3}\right)+\left(8+3 m_{2}^{2}-10 m_{3}\right) \cos 2 \theta}{24 r}+O\left(\frac{1}{r^{2}}\right) \\
\frac{f_{2}^{2}}{L^{2}} & =2 r-\frac{4+m_{2} \cos \theta}{2}+\frac{3\left(8+m_{2}^{2}-2 m_{3}\right)+\left(8+3 m_{2}^{2}-10 m_{3}\right) \cos 2 \theta}{24 r}+O\left(\frac{1}{r^{2}}\right) \\
\frac{f_{3}^{2}}{L^{2} \sin ^{2} \theta} & =1+\frac{m_{2} \cos \theta}{2 r}-\frac{3\left(m_{2}^{2}-8 m_{3}\right)+\left(32+3 m_{2}^{2}-40 m_{3}\right) \cos 2 \theta}{96 r^{2}}+O\left(\frac{1}{r^{3}}\right) \\
\frac{\rho^{2}}{L^{2}} & =\frac{1}{4 r^{2}}+\frac{m_{2} \cos \theta}{8 r^{3}}+\frac{-3\left(16+m_{2}^{2}-8 m_{3}\right)+\left(16-3 m_{2}^{2}+40 m_{3}\right) \cos 2 \theta}{384 r^{4}}+O\left(\frac{1}{r^{5}}\right)
\end{aligned}
$$

Next we present the mapping of the $(r, \theta)$ coordinates for large values of $r$ to an FG coordinate system $(u, \tilde{\theta})$ for a general bubbling solution. We need this map to define the large $r$ cut-off function as well as to perform the Kaluza-Klein (KK) reduction in the calculation of the expectation value of the stress tensor in section 4 .

It is natural to consider a Wilson surface living on $A d S_{3} \times S^{3}$. This space is related to $\mathbb{R}^{6}$ by a Weyl rescaling. We can choose to adapt our FG chart to either space; here we choose the former. The general FG metric that preserves the $A d S_{3} \times S^{3} \times S^{3}$ isometry of a bubbling solution is given by

$$
d s^{2}=L^{2}\left[\frac{4}{u^{2}}\left(d u^{2}+\alpha_{1} d s_{A d S_{3}}^{2}+\alpha_{2} d s_{S^{3}}^{2}\right)+\alpha_{3} d \tilde{\theta}^{2}+\alpha_{4} d s_{\tilde{S}^{3}}^{2}\right]
$$

Equating this metric with the bubbling metric (2.1) we find

$$
\begin{aligned}
& f_{1}^{2}=\frac{4 L^{2} \alpha_{1}}{u^{2}}, \quad f_{2}^{2}=\frac{4 L^{2} \alpha_{2}}{u^{2}}, \quad f_{3}^{2}=L^{2} \alpha_{4} \\
& 4 \rho^{2}\left(d r^{2}+r^{2} d \theta^{2}\right)=\frac{4 L^{2} d u^{2}}{u^{2}}+L^{2} \alpha_{3} d \tilde{\theta}^{2}
\end{aligned}
$$

We regulate the spacetime at a small value of $u$ and identify this with $\varepsilon$ : the (dimensionless) UV cut-off on $A d S_{3} \times S^{3}$. The boundary conditions on the coordinate map and the functions $\alpha_{i}(u, \tilde{\theta})$ at small $u$ must be chosen to ensure that the boundary metric is $d s_{A d S_{3}}^{2}+d s_{S^{3}}^{2}$ and the transverse $S^{4}$ is recovered. We find

$$
\begin{aligned}
r & =\frac{2}{u^{2}}+\ldots, \quad \theta=\tilde{\theta}+\ldots \\
\alpha_{1}=1+\ldots, \quad \alpha_{2} & =1+\ldots, \quad \alpha_{3}=1+\ldots, \quad \alpha_{4}=\sin ^{2} \tilde{\theta}+\ldots
\end{aligned}
$$


Whilst we have not been able to solve (2.22) in closed form, we can build the coordinate map as an asymptotic expansion in $u$. The mapping is given by

$$
\begin{aligned}
r= & \frac{2}{u^{2}}+\frac{m_{2} \cos \tilde{\theta}}{4}+\frac{3\left(-16-m_{2}^{2}+8 m_{3}\right)+\left(16-21 m_{2}^{2}+40 m_{3}\right) \cos 2 \tilde{\theta}}{768} u^{2} \\
& +\frac{\cos \tilde{\theta}}{18432}\left(48 m_{2}-43 m_{2}^{3}+40 m_{2} m_{3}+80 m_{4}\right. \\
& \left.-\left(48 m_{2}-203 m_{2}^{3}+680 m_{2} m_{3}-560 m_{4}\right) \cos 2 \tilde{\theta}\right) u^{4}+O\left(u^{6}\right)
\end{aligned}
$$

and

$$
\begin{aligned}
\theta= & \tilde{\theta}-\frac{m_{2} \sin \tilde{\theta}}{8} u^{2}-\frac{\left(16-27 m_{2}^{2}+40 m_{3}\right) \cos \tilde{\theta} \sin \tilde{\theta}}{768} u^{4} \\
& +\frac{\sin \tilde{\theta}}{18432}\left(296 m_{2} m_{3}-48 m_{2}-98 m_{2}^{3}-200 m_{4}\right. \\
& \left.+\left(48 m_{2}-139 m_{2}^{3}+400 m_{2} m_{3}-280 m_{4}\right) \cos 2 \tilde{\theta}\right) u^{6}+O\left(u^{8}\right)
\end{aligned}
$$

The area integral and action integral both diverge at large $r$. It is useful to express the coordinate map as a cut-off relation $r_{c}=r_{c}(\theta, \varepsilon)$. This is found by first inverting the relation (2.25) in the small $u$ limit and then eliminating $\tilde{\theta}$ from (2.24). The result is

$$
\begin{aligned}
r_{c}(\theta, \varepsilon)= & \frac{2}{\varepsilon^{2}}+\frac{m_{2} \cos \theta}{4}+\frac{-3\left(16+5 m_{2}^{2}-8 m_{3}\right)+\left(16-9 m_{2}^{2}+40 m_{3}\right) \cos 2 \theta}{768} \varepsilon^{2} \\
& +\frac{\cos \theta}{9216}\left(-48 m_{2}+55 m_{2}^{3}-160 m_{2} m_{3}+40 m_{4}\right. \\
& \left.+\left(48 m_{2}+25 m_{2}^{3}-160 m_{2} m_{3}+280 m_{4}\right) \cos 2 \theta\right) \varepsilon^{4}+O\left(\varepsilon^{6}\right)
\end{aligned}
$$

\section{Holographic entanglement entropy}

The Ryu-Takayanagi prescription [3, 4] states that the entanglement entropy of a spatial region $\mathcal{A}$ is given by the area of a co-dimension two minimal surface $\mathcal{M}$ in the bulk that is anchored on the AdS boundary at $\partial \mathcal{A}$ :

$$
S_{\mathcal{A}}=\frac{A_{\min }}{4 G_{N}^{(11)}}
$$

Since we are dealing with static states of our CFT, this surface lies on a constant time slice. If this surface is not unique, we choose the one whose area is minimal among all such surfaces homologous to $\mathcal{A} .^{2}$

In the following section we derive the minimal surface $\mathcal{M}$ for a general bubbling solution and show that its restriction to the boundary maps to a four-sphere in $\mathbb{R}^{6}$. We then evaluate its regulated area and compare with our expectations from $\mathbb{R}^{6}$.

\footnotetext{
${ }^{2}$ This minimal surface prescription was recently established on a firm footing by the analysis of [38].
} 


\subsection{Minimal surface geometry}

A bubbling geometry is an $A d S_{3} \times S^{3} \times S^{3}$ fibration over $\Sigma$. We consider a surface $\mathcal{M}$ at constant $t$ that fills the $S^{3} \times S^{3}$ and has profile $z=z(w, \bar{w}, l)$, where $z$ is the $A d S_{3}$ radial coordinate defined in (2.2). The area functional becomes

$$
A(\mathcal{M})=2 \operatorname{Vol}\left(S^{3}\right)^{2} \int d l \int_{\Sigma} d^{2} w \frac{f_{1} f_{2}^{3} f_{3}^{3} \rho^{2}}{z} \sqrt{1+\frac{f_{1}^{2}}{z^{2} \rho^{2}} \frac{\partial z}{\partial w} \frac{\partial z}{\partial \bar{w}}+\left(\frac{\partial z}{\partial l}\right)^{2}}
$$

The equations of motion derived from this functional are solved by

$$
z(w, \bar{w}, l)^{2}+l^{2}=R^{2}
$$

This semicircle is simply a co-dimension two minimal surface in $A d S_{3}$. Following $[12,13]$ it is straightforward to see that this is in fact the surface of minimal area (within this ansatz).

The surface (3.3) is independent of the $A d S_{7}$ radial coordinate. Thus, the boundary $\partial \mathcal{A}$ of the entangling region on $A d S_{3} \times S^{3}$ is given by the same formula. To understand this, let us consider two coordinate charts on $\mathbb{R}^{6}$ :

$$
d s_{\mathbb{R}^{6}}^{2}=z^{2}\left(\frac{d z^{2}+d t^{2}+d l^{2}}{z^{2}}+d s_{S^{3}}^{2}\right)=d t^{2}+d \bar{r}^{2}+\bar{r}^{2}\left(d \chi^{2}+\sin ^{2} \chi d s_{S^{3}}^{2}\right)
$$

The map between these two charts is given by

$$
z=\bar{r} \sin \chi, \quad l=\bar{r} \cos \chi
$$

Thus, our $\partial \mathcal{A}$ on $A d S_{3} \times S^{3}$ can be written as a four-sphere of radius $R$ on $\mathbb{R}^{6}$ (given by $\bar{r}=R$ ) after a Weyl rescaling.

\subsection{Evaluating the area integral}

The combination of metric factors that appears in the area integral (3.2) can be written

$$
f_{1} f_{2}^{3} f_{3}^{3} \rho^{2}=\frac{1}{4}\left|\partial_{w} h\right|^{2} h W_{-}
$$

The entanglement entropy is proportional to the area evaluated on the surface (3.3):

$$
S_{\mathcal{A}}=\frac{\operatorname{Vol}\left(S^{3}\right)^{2}}{8 G_{N}^{(11)}} \int d l \frac{R}{R^{2}-l^{2}}\left(J_{1}+J_{2}\right)
$$

where we have defined

$$
\begin{aligned}
& J_{1} \equiv \int_{\Sigma} d^{2} w\left|\partial_{w} h\right|^{2} h|G-\bar{G}| \\
& J_{2} \equiv-2 \int_{\Sigma} d^{2} w\left|\partial_{w} h\right|^{2} h|G|^{2}
\end{aligned}
$$

Substituting $w=r e^{i \theta}$ into (2.12) we find for $J_{1}$

$$
J_{1}=-4 L^{9} \sum_{i=1}^{2 n}(-1)^{i} \int_{0}^{\pi} d \theta \sin \theta \int_{0}^{r_{c}(\theta, \varepsilon)} d r \frac{r^{2}\left(r \cos \theta-\xi_{i}\right)}{\sqrt{r^{2}+\xi_{i}^{2}-2 r \xi_{i} \cos \theta}}
$$


The overall minus sign follows from the fact that $G-\bar{G}<0$ on the upper half-plane. We carefully evaluate this expression in appendix A.1. The final result is given in equation (A.5) and takes the form

$$
J_{1}=L^{9}\left[\frac{64}{3 \varepsilon^{4}}+\frac{-24+3 m_{2}^{2}-8 m_{3}}{15}+O\left(\varepsilon^{2}\right)\right]
$$

Next we consider the second term

$$
\begin{aligned}
J_{2}= & -2 \int_{\Sigma} d^{2} w\left|\partial_{w} h\right|^{2} h|G|^{2} \\
= & -2 L^{9} \int_{0}^{\pi} d \theta \sin \theta \int_{0}^{r_{c}(\theta, \varepsilon)} d r r^{2} \\
& \times\left\{2 n+2 \sum_{i<j}(-1)^{i+j} \frac{r^{2}-r \cos \theta\left(\xi_{i}+\xi_{j}\right)+\xi_{i} \xi_{j}}{\sqrt{r^{2}-2 r \xi_{i} \cos \theta+\xi_{i}^{2}} \sqrt{r^{2}-2 r \xi_{j} \cos \theta+\xi_{j}^{2}}}\right\}
\end{aligned}
$$

We carefully evaluate this integral in appendix A.2 and the final result is (A.22)

$$
J_{2}=L^{9}\left[-\frac{64}{3 \varepsilon^{2}}-\frac{4}{3} \sum_{i<j}(-1)^{i+j}\left|\xi_{i}-\xi_{j}\right|^{3}+O\left(\varepsilon^{2}\right)\right]
$$

Note that the second term cannot be expressed in terms of the moments $m_{k}$.

Now we handle the integral over $l$. Recall that the minimal surface formula (3.3) describes a semicircle for which $z \in[0, R]$ and $l \in[-R, R]$. Note that $J_{1,2}$ are independent of $l$ because the cut-off function is. The $l$ integral diverges at both limits; rewriting via (3.3) as an integral over $z$, we regulate with a cut-off at $z=\eta$ :

$$
\begin{aligned}
\int_{-\sqrt{R^{2}-\eta^{2}}}^{\sqrt{R^{2}-\eta^{2}}} d l \frac{R}{R^{2}-l^{2}} & =2 \int_{0}^{\sqrt{R^{2}-\eta^{2}}} d l \frac{R}{R^{2}-l^{2}}=2 \int_{\eta}^{R} d z \frac{R}{z \sqrt{R^{2}-z^{2}}} \\
& =2 \log \left(\frac{R+\sqrt{R^{2}-\eta^{2}}}{\eta}\right)=2 \log \left(\frac{2 R}{\eta}\right)-\frac{\eta^{2}}{2 R^{2}}+O\left(\eta^{4}\right)
\end{aligned}
$$

Finally we put these pieces together to compute the divergent entanglement entropy (3.7):

$$
\begin{aligned}
S_{\mathcal{A}}= & \frac{L^{9} \operatorname{Vol}\left(S^{3}\right)^{2}}{4 G_{N}^{(11)}}\left[\frac{64}{3 \varepsilon^{4}}-\frac{64}{3 \varepsilon^{2}}+\frac{-24+3 m_{2}^{2}-8 m_{3}}{15}\right. \\
& \left.-\frac{4}{3} \sum_{i<j}(-1)^{i+j}\left|\xi_{i}-\xi_{j}\right|^{3}+O\left(\varepsilon^{2}\right)\right] \log \left(\frac{2 R}{\eta}\right)
\end{aligned}
$$

Employing the definitions

$$
L=(\pi N)^{1 / 3} \ell_{P}, \quad 8 \pi G_{N}^{(11)}=2^{7} \pi^{8} \ell_{P}^{9}, \quad \operatorname{Vol}\left(S^{3}\right)=2 \pi^{2}
$$

this becomes

$$
\begin{aligned}
S_{\mathcal{A}}= & \frac{4 N^{3}}{3}\left[\frac{1}{\varepsilon^{4}}-\frac{1}{\varepsilon^{2}}+\frac{-24+3 m_{2}^{2}-8 m_{3}}{320}\right. \\
& \left.-\frac{1}{16} \sum_{i<j}(-1)^{i+j}\left|\xi_{i}-\xi_{j}\right|^{3}+O\left(\varepsilon^{2}\right)\right] \log \left(\frac{2 R}{\eta}\right)
\end{aligned}
$$


Evaluating this result on the vacuum we find

$$
S_{\mathcal{A}}^{(0)}=\frac{4 N^{3}}{3}\left[\frac{1}{\varepsilon^{4}}-\frac{1}{\varepsilon^{2}}+\frac{3}{8}+O\left(\varepsilon^{2}\right)\right] \log \left(\frac{2 R}{\eta}\right)
$$

Subtracting this vacuum contribution from (3.16) we arrive at our final result for the change in entanglement entropy due to the presence of the Wilson surface:

$$
\Delta S_{\mathcal{A}}=\frac{4 N^{3}}{3}\left[\frac{16+3 m_{2}^{2}-8 m_{3}}{320}-\frac{1}{16} \sum_{i<j}(-1)^{i+j}\left|\xi_{i}-\xi_{j}\right|^{3}-\frac{1}{2}\right] \log \left(\frac{2 R}{\eta}\right)
$$

Note that the power divergences with respect to the FG cut-off $\varepsilon$ are cancelled in this subtraction and only a logarithmic divergence in $\eta$ remains.

\subsection{Physical interpretation}

In this section we give a physical interpretation of our result for the entanglement entropy. First, recall that during the calculation we introduced two separate regulators. The FG cutoff $\varepsilon$ can be viewed as a regular UV cut-off for a holographic theory with a six-dimensional $A d S_{3} \times S^{3}$ boundary. In addition, when performing the integral over the $A d S_{3}$ coordinate $l$ in (3.13) we introduced a cut-off $\eta$ on the $A d S_{3}$ radial coordinate $z$ in Poincare slicing (2.2). One might be tempted to view $\eta$ as purely an IR cut-off that regulates the infinite volume of the boundary theory. However, it also has an interpretation as a UV cut-off on the minimal distance to the Wilson surface in the boundary theory.

This interpretation is most easily demonstrated by considering the vacuum spacetime. The map that relates $A d S_{7} \times S^{4}$ with $A d S_{3} \times S^{3}$ boundary in (2.9) to a metric with $\mathbb{R}^{6}$ boundary

$$
d s^{2}=\frac{4 L^{2}}{\tilde{u}^{2}}\left(d \tilde{u}^{2}+d t^{2}+d l^{2}+d r^{2}+r^{2} d s_{S^{3}}^{2}\right)+L^{2}\left(d q^{2}+\sin ^{2} q d s_{\tilde{S}^{3}}^{2}\right)
$$

is given by

$$
z=\tilde{u} \cosh p \quad r=\tilde{u} \sinh p
$$

Setting $\tilde{u}=\tilde{\varepsilon}$ imposes a (dimensionful) holographic UV cut-off on the theory living on the $\mathbb{R}^{6}$ boundary. Since the minimal value of the coordinate $p$ is zero, it follows from (3.20) that the range of $z$ is bounded by $z>\tilde{\varepsilon}$ and hence the $A d S_{3}$ cut-off $\eta$ is related to the uniform UV cut-off $\tilde{\varepsilon}$.

At this point we do not have an analog of the map (3.20) for the bubbling solution that is valid for all values of the coordinates. We can construct the map for the asymptotic region defined by the FG expansion for the $A d S_{3} \times S^{3}$ boundary theory (2.21), however this expansion breaks down once the FG coordinate $u$ is not small. As discussed in [13] one can construct a map that is valid also near $z=0$ by patching together the expansion near the $A d S_{3}$ boundary and the FG boundary. We will not pursue this construction here since we focus all our calculations on the theory living on the $A d S_{3} \times S^{3}$ boundary. However, since the metric is asymptotically AdS, we expect that one should obtain only a small 
modification to the identification of the UV cut-offs that, crucially, does not affect the logarithmically divergent term in the entanglement entropy.

Physically, the interpretation of $\eta$ as a UV cut-off and the form of the subtracted entanglement entropy (3.18) is quite natural. Note that the dominant contributions to the entanglement entropy come from UV degrees of freedom located near the entangling surface. Since the Wilson surface (at fixed time) intersects the entangling surface at two points in our geometry (see figure 1), the defect contribution to the entanglement entropy has essentially the same dimensionality as the entanglement entropy of a two-dimensional CFT. This is also reflected in the $A d S_{3}$ slicing we employ and the fact that the minimal surface we find in the bulk (3.3) is familiar from $A d S_{3} / \mathrm{CFT}_{2}$. Hence an argument along the lines of those given in [13] shows that the extra divergent contribution of the Wilson surface should be logarithmic, wherein the cut-off is associated with a minimal distance to the defect.

\section{Holographic stress tensor}

The goal of the present section is to calculate the one point function of the six-dimensional stress tensor holographically for an asymptotically $A d S_{7} \times S^{4}$ bubbling solution. Since the bubbling solutions are eleven-dimensional one has to utilize the machinery of KK holography that was developed in [39]. Note that a similar calculation was performed in [23] for the type IIB bubbling solution dual to half-BPS Wilson loop defects and we will largely adopt their method to our case.

The KK reduction of eleven-dimensional supergravity on $S^{4}$ produces a sevendimensional supergravity (with negative cosmological constant) with infinite towers of massive fields [40, 41]. These can be classified by their seven-dimensional spin and representation of the relevant $\mathrm{SO}(5)$ spherical harmonics (scalar and tensorial) on $S^{4}$. One difficulty is the mixing of modes coming from the eleven-dimensional supergravity as well as the fact that seven-dimensional fields can be related by eleven-dimensional diffeomorphisms, leading to nonlinear gauge symmetries. The resulting seven-dimensional action can be diagonalized and the masses of all the fields were determined in [40-42].

Via the AdS/CFT correspondence the seven-dimensional supergravity fields are dual to operators in the six-dimensional $(2,0)$-theory. The precise dictionary can be found for example in [43].

In [39] it was argued that in order to obtain a local seven-dimensional supergravity action without higher derivatives one needs in general to perform a KK reduction map that is nonlinear and relates the eleven- and seven-dimensional fields schematically as

$$
\Psi_{7}=\psi_{11}+\mathcal{K} \psi_{11} \psi_{11}+\ldots
$$

Here, $\mathcal{K}$ is a differential operator and the ellipsis denotes higher order terms with three or more eleven-dimensional fields. This nonlinear mixing in general complicates holographic calculations (see e.g. [23]). However, a simple rule was derived in [39] to determine when and which nonlinear terms appear. For a supergravity field dual to a dimension $\Delta$ operator in the CFT, the only nonlinear terms that can appear are the ones for which the sum of 
the dimensions of their respective dual operators is less than or equal to $\Delta$. When this rule is applied to the stress tensor, which has $\Delta=6$, it is clear that there can be no nonlinear mixing since the operators with lowest dimension have $\Delta=4 .^{3}$

The starting point for the calculation of the holographic stress tensor is to decompose the eleven-dimensional metric into a $A d S_{7} \times S^{4}$ part and a perturbation, denoted as $g^{(0)}$ and $h$ respectively:

$$
d s^{2}=g_{M N} d x^{M} d x^{N}=\left(g_{M N}^{(0)}+h_{M N}\right) d x^{M} d x^{N} .
$$

We use the FG coordinate chart (2.21) where we can identify $\tilde{\theta}$ as the polar angle on $S^{4}$. The Wilson surface preserves an $\mathrm{SO}(4)$ subgroup of the R-symmetry and so does the bubbling geometry. Therefore, performing the harmonic decomposition on the eleven-dimensional fields we obtain contributions only from spherical harmonics invariant under $\mathrm{SO}(4)$. These depend only on the polar angle $\tilde{\theta}$. The zero mode on $S^{4}$ of an eleven-dimensional field that only has nontrivial dependence on $\tilde{\theta}$ can be expressed as

$$
\bar{\phi}(x)=\frac{\int_{0}^{\pi} d \tilde{\theta} \phi(x, \tilde{\theta}) \sin ^{3} \tilde{\theta}}{\int_{0}^{\pi} d \tilde{\theta} \sin ^{3} \tilde{\theta}}
$$

The reduced seven-dimensional metric, which satisfies the seven-dimensional linearized Einstein equation, is given by the following combination ${ }^{4}$

$$
d s_{7}^{2}=\left[\left(1+\frac{1}{5} \bar{\pi}\right) g_{\mu \nu}^{(0)}+\bar{h}_{\mu \nu}\right] d x^{\mu} d x^{\nu}
$$

Here, $g^{(0)}$ is the $A d S_{7}$ vacuum metric whereas $\bar{h}_{\mu \nu}$ is the zero mode of the fluctuations in the seven dimensions. The field $\bar{\pi}$ is the zero mode of the trace of the fluctuations of the metric along the $S^{4}$ directions:

$$
\pi(x, \tilde{\theta}) \equiv h^{a b} g_{a b}^{(0)}
$$

The factor of $\frac{1}{5}$ in (4.4) comes from a Weyl rescaling to bring the KK reduced metric to the Einstein frame in seven dimensions.

The calculation of the one point function of the stress tensor from the sevendimensional metric (4.4) utilizes the standard method of holographic renormalization. In our case $\bar{\pi}$ vanishes, and consequently the reduced metric is already in the FG form

$$
d s_{7}^{2}=\frac{4 L^{2}}{u^{2}}\left(d u^{2}+g_{i j} d x^{i} d x^{j}\right)
$$

where the large $r$ limit corresponds to $u \rightarrow 0$ and the metric $g_{i j}$ can be expressed as a power series in $u$. The holographic stress tensor can then be calculated immediately using the formulae for $d=6$ given in [44]. For the convenience of the reader and completeness

\footnotetext{
${ }^{3}$ There is a 'doubleton' field dual to an operator of dimension $\Delta=2$, but such fields are free and decouple from the dynamics.

${ }^{4}$ Our index conventions are: $M, N, \ldots$ are eleven-dimensional indices, $\mu, \nu, \ldots$ are $A d S_{7}$ indices and $a, b, \ldots$ are $S^{4}$ indices.
} 
we present the details of these calculations in appendix B. We have also checked that the counter-term approach developed in [45] gives the same result for the stress tensor. The final result for the change in the expectation value of the stress tensor in the presence of the Wilson surface on $A d S_{3} \times S^{3}$ is

$$
\Delta\left\langle T_{i j}\right\rangle d x^{i} d x^{j}=\frac{N^{3}}{160 \pi^{3}}\left(16+3 m_{2}^{2}-8 m_{3}\right)\left(d s_{A d S_{3}}^{2}-d s_{S^{3}}^{2}\right)
$$

Note that the dependence of $N^{3}$ is as expected from a back-reacted supergravity solution. In addition, the expression depends only on the first two nontrivial moments $m_{2}, m_{3}$ of the bubbling solution. The stress tensor contribution has a form that respects the $s o(2,2 \mid \mathbb{R}) \oplus s o(4 \mid \mathbb{R})$ of the Wilson surface and is traceless in line with the absence of a conformal anomaly on $A d S_{3} \times S^{3}$. The use of this result is two-fold. First, any nontrivial holographic observable is useful to understand $(2,0)$ theory better. Second, the stress tensor expectation value is an important ingredient in the calculation of the entanglement entropy using the replica trick, as we discuss briefly in 6 .

\section{Expectation value of the Wilson surface operator}

The expectation value for the Wilson surface operator can be obtained from the following formula:

$$
\left\langle W_{\Gamma}\right\rangle=\exp \left[-\left(I-I_{(0)}\right)\right]
$$

where $I$ is the eleven-dimensional supergravity action evaluated on a general bubbling solution and $I_{(0)}$ is the action evaluated on the $A d S_{7} \times S^{4}$ vacuum. The action is given by

$$
\begin{aligned}
I= & \frac{1}{8 \pi G_{N}^{(11)}}\left[\frac{1}{2} \int d^{11} x \sqrt{g}\left(R-\frac{1}{48} F_{M N P Q} F^{M N P Q}\right)-\frac{1}{12} \int C \wedge F \wedge F\right. \\
& \left.+\int d^{10} x \sqrt{\gamma} K\right]
\end{aligned}
$$

The final term is the Gibbons-Hawking boundary term, which is necessary to make the variational principle well-defined for spacetimes with boundary. The metric functions for the bubbling solution are given in (2.5) and the four-form field strength is given by

$$
F=\left(f_{1}\right)^{3} g_{1 m} \omega_{A d S_{3}} \wedge e^{m}+\left(f_{2}\right)^{3} g_{2 m} \omega_{S^{3}} \wedge e^{m}+\left(f_{3}\right)^{3} g_{3 m} \omega_{\tilde{S}^{3}} \wedge e^{m}
$$

where $e^{m}$ are the vielbeins on the Riemann surface $\Sigma, \omega_{X}$ denotes the volume form for a unit-radius space $X$ and the expressions for $g_{I m}$ with $I=1,2,3$ can be found in appendix C.

\subsection{Action as a total derivative}

First we demonstrate the well-known fact that the on-shell action of eleven-dimensional supergravity is a total derivative. Using the Einstein equation, the Ricci scalar can be eliminated from the bulk term of the on-shell supergravity action (5.2):

$$
I_{\text {bulk }}=\frac{1}{16 \pi G_{N}^{(11)}} \int\left(-\frac{1}{3} F \wedge * F-\frac{1}{6} C \wedge F \wedge F\right)
$$


The equation of motion can be expressed as

$$
d * F+\frac{1}{2} F \wedge F=0
$$

The first term in the action can then be written as

$$
\begin{aligned}
F \wedge * F & =d C \wedge * F \\
& =d(C \wedge * F)+C \wedge d * F \\
& =d(C \wedge * F)-\frac{1}{2} C \wedge F \wedge F
\end{aligned}
$$

Plugging this expression into (5.4) the $C \wedge F \wedge F$ terms cancel and the on-shell value of the action is indeed a total derivative:

$$
I_{\text {bulk }}=-\frac{1}{48 \pi G_{N}^{(11)}} \int d(C \wedge * d C)
$$

The duals of the three contributions to the field strength are given by

$$
\begin{aligned}
& * F_{(1)}=\frac{f_{2}^{3} f_{3}^{3}}{f_{1}^{3}}\left(r \partial_{r} b_{1} d \theta-\frac{1}{r} \partial_{\theta} b_{1} d r\right) \wedge \omega_{S^{3}} \wedge \omega_{\tilde{S}^{3}} \\
& * F_{(2)}=\frac{f_{1}^{3} f_{3}^{3}}{f_{2}^{3}}\left(r \partial_{r} b_{2} d \theta-\frac{1}{r} \partial_{\theta} b_{2} d r\right) \wedge \omega_{A d S_{3}} \wedge \omega_{\tilde{S}_{3}} \\
& * F_{(3)}=\frac{f_{1}^{3} f_{2}^{3}}{f_{3}^{3}}\left(-r \partial_{r} b_{3} d \theta+\frac{1}{r} \partial_{\theta} b_{3} d r\right) \wedge \omega_{A d S_{3}} \wedge \omega_{S^{3}}
\end{aligned}
$$

Using the expression for the metric functions $f_{i}$ from (2.5), the ratios appearing in (5.8) can be expressed as

$$
\begin{aligned}
& \left(\frac{f_{2} f_{3}}{f_{1}}\right)^{3}=\frac{h W_{-}^{2}}{4 W_{+}\left(1-|G|^{2}\right)} \\
& \left(\frac{f_{1} f_{3}}{f_{2}}\right)^{3}=\frac{h W_{+}^{2}}{4 W_{-}\left(1-|G|^{2}\right)} \\
& \left(\frac{f_{1} f_{2}}{f_{3}}\right)^{3}=\frac{16 h\left(1-|G|^{2}\right)^{2}}{W_{+} W_{-}}
\end{aligned}
$$

Since the volume forms on the $A d S_{3}$ and the three-spheres are all closed, the bulk action (5.7) reduces to a total derivative over the two-dimensional Riemann surface $\Sigma$. In terms of the polar coordinates $r, \theta$, which were introduced in section 2.1 by setting $w=r e^{i \theta}$, the bulk part of the action can be written as follows:

$$
\begin{aligned}
I_{\text {bulk }} & =-\frac{1}{48 \pi G_{N}^{(11)}} \int d(C \wedge * F) \\
& =-\frac{1}{48 \pi G_{N}^{(11)}} \int\left(\partial_{r} a_{r}+\partial_{\theta} a_{\theta}\right) d r \wedge d \theta \wedge \omega_{A d S_{3}} \wedge \omega_{S^{3}} \wedge \omega_{\tilde{S}^{3}}
\end{aligned}
$$




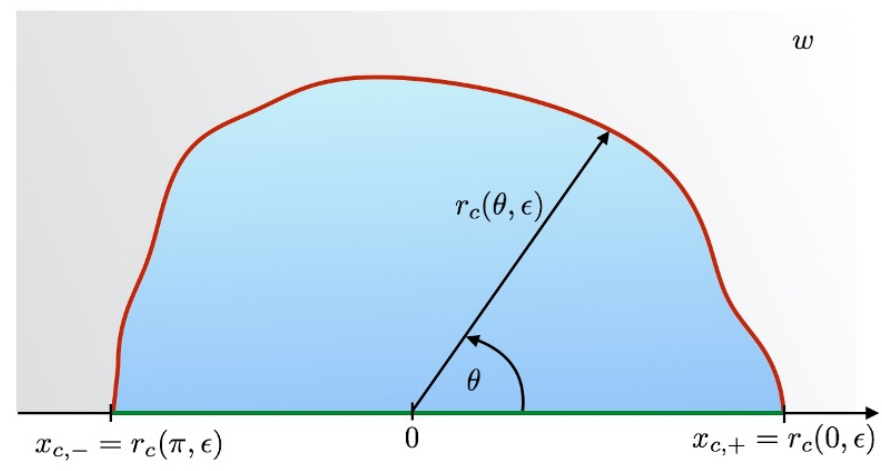

Figure 4. The two boundary components: the cut-off surface (red) and the boundary along $x \equiv \operatorname{Re} w$ (green).

where $a_{r}$ and $a_{\theta}$ are given by

$$
\begin{aligned}
& a_{r}=-\frac{f_{2}^{3} f_{3}^{3}}{2 f_{1}^{3}} r \partial_{r}\left(b_{1}^{2}\right)+\frac{f_{1}^{3} f_{3}^{3}}{2 f_{2}^{3}} r \partial_{r}\left(b_{2}^{2}\right)+\frac{f_{1}^{3} f_{2}^{3}}{2 f_{3}^{3}} r \partial_{r}\left(b_{3}^{2}\right) \\
& a_{\theta}=-\frac{f_{2}^{3} f_{3}^{3}}{2 f_{1}^{3}} \frac{1}{r} \partial_{\theta}\left(b_{1}^{2}\right)+\frac{f_{1}^{3} f_{3}^{3}}{2 f_{2}^{3}} \frac{1}{r} \partial_{\theta}\left(b_{2}^{2}\right)+\frac{f_{1}^{3} f_{2}^{3}}{2 f_{3}^{3}} \frac{1}{r} \partial_{\theta}\left(b_{3}^{2}\right)
\end{aligned}
$$

We have shown that the on-shell action can be written as a boundary term: the bulk term reduces to a total derivative and the Gibbons-Hawking term is also boundary term. The boundary in the $w$ plane has two pieces (see figure 4). First one has the cut-off surface parametrized by $r_{c}(\theta, \varepsilon)$, where $\varepsilon$ is the FG cut-off. Second one has the real line, which is parametrized in $r, \theta$ coordinates by $r \in\left[0, r_{c}(0, \pi, \varepsilon)\right]$ and $\theta=0, \pi$, respectively. In the following we will evaluate the contribution from each piece in turn.

\subsection{Gibbons-Hawking term}

The Gibbons-Hawking term in (5.2) is the boundary term that has to be added to the action in order to give a well-defined gravitational variational principle. A ten-dimensional surface defined by a constraint

$$
F\left(x^{M}\right)=0
$$

can be parameterized by a set of coordinates $\sigma^{\alpha}$. The induced metric is defined via

$$
\gamma_{\alpha \beta}=e_{\alpha}^{M} e_{\beta}^{N} g_{M N}, \quad e_{\alpha}^{M}=\frac{\partial x^{M}}{\partial \sigma^{\alpha}}
$$

and its determinant is given by

$$
\sqrt{\gamma}=\frac{1}{z^{3}} 2 \rho f_{1}^{3} f_{2}^{3} f_{3}^{3} \omega_{S^{3}} \omega_{\tilde{S}^{3}}
$$

The normal vector (which we assume to be always space-like) is defined via

$$
\hat{n}_{M}=\frac{1}{\sqrt{\frac{\partial F}{\partial x^{N}} \frac{\partial F}{\partial x^{P}} g^{N P}}} \frac{\partial F}{\partial x^{M}}
$$


The extrinsic curvature and its trace are defined via

$$
K_{\alpha \beta}=\left(\nabla_{M} \hat{n}_{N}\right) e_{\alpha}^{M} e_{\beta}^{N}, \quad K=\gamma^{\alpha \beta} K_{\alpha \beta}
$$

As discussed above the boundary has two components, which we now study in turn.

\subsubsection{Real line contribution}

The real line is defined by $F=y=0$, where $w=x+i y$, and the induced metric is simply obtained by dropping the $g_{y y}$ component of the eleven-dimensional metric. The normal vector is given by

$$
\hat{n}^{M}=\frac{1}{2 \rho} \delta_{y}^{M}
$$

Using the form of the metric it is straightforward to determine the trace of the extrinsic curvature

$$
K=\frac{1}{L^{3}}\left(\frac{3 \partial_{y} f_{1}}{2 \rho f_{1}}+\frac{3 \partial_{y} f_{2}}{2 \rho f_{2}}+\frac{3 \partial_{y} f_{3}}{2 \rho f_{3}}+\frac{\partial_{y} \rho}{2 \rho^{2}}\right)
$$

The Gibbons-Hawking term along the real line can be determined from the expansion of the metric functions in the $y \rightarrow 0$ limit given. This can be obtained from the formulae in appendix D. We do not present the details of this calculation but just present the final result which is

$$
\int d^{10} x \sqrt{\gamma} K=\lim _{y \rightarrow 0} \operatorname{Vol}\left(S^{3}\right)^{2} \operatorname{Vol}\left(A d S_{3}\right) L^{9} \int d x\left[24 y^{2}+O\left(y^{3}\right)\right]=0
$$

which vanishes. This result was expected since at any point on the real line, one of the three-spheres shrinks to zero size and the space closes off.

\subsubsection{Large $r$ contribution}

In this section we determine the contribution of the Gibbons-Hawking term from the large $r$ cut-off surface defined by the equation

$$
F(r, \theta)=r-r_{c}(\theta, \varepsilon)=0
$$

for small $\varepsilon$, where $r_{c}(\theta, \varepsilon)$ is given by $(2.26)$. Hence the surface extends along $A d S_{3}$ and the two three-spheres and the induced metric in these directions is identical to the metric. We choose to use $\theta$ to parametrize the cut-off surface and find the following nontrivial components of the vielbein $e_{\alpha}^{M}$ :

$$
e_{\beta}^{\alpha}=\delta_{\beta}^{\alpha}, \quad e_{\theta}^{r}=\partial_{\theta} r_{c}(\theta, \varepsilon)
$$


Using the formulae (5.15) and (5.16) we can calculate $\sqrt{\gamma} K$ and expand the result in a power series in $\varepsilon$ :

$$
\begin{aligned}
\sqrt{\gamma} K= & L^{9} \sin ^{3} \theta\left\{\frac{192}{\varepsilon^{6}}+\frac{96 m_{2} \cos \theta}{\varepsilon^{4}}\right. \\
& +\frac{-3\left(16-3 m_{2}^{2}-40 m_{3}\right)+5 \cos 2 \theta\left(16+3 m_{2}^{2}+40 m_{3}\right)}{8 \varepsilon^{2}} \\
& \frac{1}{4} \cos \theta\left[-8 m_{2}+m_{2}^{3}-6 m_{2} m_{3}+10 m_{4}\right. \\
& \left.\left.+\cos 2 \theta\left(24 m_{2}+m_{2}^{3}-10 m_{2} m_{3}+70 m_{4}\right)\right]\right\}+O\left(\varepsilon^{2}\right)
\end{aligned}
$$

After performing the integral over $\theta$ we find that the Gibbons-Hawking term is determined completely by the contribution at large $r$, namely

$$
I_{\mathrm{GH}} \equiv \frac{1}{8 \pi G_{N}^{(11)}} \int d^{10} x \sqrt{\gamma} K=\frac{L^{9}}{8 \pi G_{N}^{(11)}} \operatorname{Vol}\left(S^{3}\right)^{2} \operatorname{Vol}\left(A d S_{3}\right)\left[\frac{256}{\varepsilon^{6}}-\frac{16}{\varepsilon^{2}}+O\left(\varepsilon^{2}\right)\right]
$$

\subsection{Bulk supergravity action}

The bulk part of the eleven-dimensional action integral (5.10) can be reduced to the following form:

$$
\begin{aligned}
I & =-\frac{1}{48 \pi G_{N}^{(11)}} \operatorname{Vol}\left(S^{3}\right)^{2} \operatorname{Vol}\left(A d S_{3}\right) \int d \theta \int d r\left(\partial_{r} a_{r}+\partial_{\theta} a_{\theta}\right) \\
& =-\frac{1}{48 \pi G_{N}^{(11)}} \operatorname{Vol}\left(S^{3}\right)^{2} \operatorname{Vol}\left(A d S_{3}\right) \oint_{\partial \Sigma(\varepsilon)} d \tau\left(\hat{n}_{r} a_{r}+\hat{n}_{\theta} a_{\theta}\right)
\end{aligned}
$$

Here $\operatorname{Vol}\left(A d S_{3}\right)$ is the (regularized) volume of $A d S_{3}$ since we consider the field theory on $A d S_{3} \times S^{3}$ and $\hat{n}_{i}$ is the unit outward normal. If a boundary component is parametrized by $r(\tau), \theta(\tau)$ then the unit outward normal vector is defined as

$$
\hat{n}_{r}=\frac{d \theta}{d \tau} d \tau, \quad \hat{n}_{\theta}=-\frac{d r}{d \tau} d \tau
$$

There are three components to the boundary:

$$
\begin{aligned}
& \partial \Sigma_{1}: \theta \in[0, \pi], r=r_{c}(\theta, \varepsilon) \\
& \partial \Sigma_{2}: \theta=0, r \in\left[0, r_{c}(0, \varepsilon)\right] \\
& \partial \Sigma_{3}: \theta=\pi, r \in\left[0, r_{c}(\pi, \varepsilon)\right]
\end{aligned}
$$

The contribution of $\partial \Sigma_{1}$ corresponds to the cut-off surface, which we parametrize by $\theta \in$ $[0, \pi]$ and $r=r_{c}(\theta, \varepsilon)$ as in section 5.2. The $\theta$ dependence of $r$ leads to an additional contribution to the normal vector

$$
\begin{aligned}
I_{(\mathrm{cut})} & =-\frac{1}{48 \pi G_{N}^{(11)}} \operatorname{Vol}\left(S^{3}\right)^{2} \operatorname{Vol}\left(A d S_{3}\right) \oint_{\partial \Sigma_{1}(\varepsilon)} d \tau\left(\hat{n}_{r} a_{r}+\hat{n}_{\theta} a_{\theta}\right) \\
& =-\left.\frac{1}{48 \pi G_{N}^{(11)}} \operatorname{Vol}\left(S^{3}\right)^{2} \operatorname{Vol}\left(A d S_{3}\right) \int_{0}^{\pi} d \theta\left(a_{r}-\frac{\partial r_{c}}{\partial \theta} a_{\theta}\right)\right|_{r=r_{c}(\theta, \varepsilon)}
\end{aligned}
$$


The integrations over $\partial \Sigma_{2}$ and $\partial \Sigma_{3}$ combine to give the integration over the real line. Putting the two together and going back to the half-plane coordinates we find

$$
\oint_{\partial \Sigma_{2}(\varepsilon)+\partial \Sigma_{3}(\varepsilon)} d \tau\left(\hat{n}_{r} a_{r}+\hat{n}_{\theta} a_{\theta}\right)=-\left.\int_{0}^{r_{c}(\theta=0, \varepsilon)} d r \frac{1}{r} a_{\theta}\right|_{\theta=0}+\left.\int_{0}^{r_{c}(\theta=\pi, \varepsilon)} d r \frac{1}{r} a_{\theta}\right|_{\theta=\pi}
$$

Using $\left.\frac{1}{r} \partial_{\theta}\right|_{\theta=0}=\partial_{y}$ and $\left.\frac{1}{r} \partial_{\theta}\right|_{\theta=\pi}=-\partial_{y}$ and the expressions for $a_{r}$ and $a_{\theta}$ given in (5.11), the contribution from the real line becomes

$$
I_{(x)}=\frac{1}{48 \pi G_{N}^{(11)}} \operatorname{Vol}\left(S^{3}\right)^{2} \operatorname{Vol}\left(A d S_{3}\right) \int_{r_{c}(\theta=\pi, \varepsilon)}^{r_{c}(\theta=0, \varepsilon)} d x\left(-\frac{f_{2}^{3} f_{3}^{3}}{2 f_{1}^{3}} \partial_{y}\left(b_{1}^{2}\right)+\frac{f_{1}^{3} f_{3}^{3}}{2 f_{2}^{3}} \partial_{y}\left(b_{2}^{2}\right)+\frac{f_{1}^{3} f_{2}^{3}}{2 f_{3}^{3}} \partial_{y}\left(b_{3}^{2}\right)\right)
$$

\subsubsection{Large $r$ contribution}

Using the expansion of $a_{r}$ and $a_{\theta}$ at large $r$ one finds the integrand of (5.27) can be written as

$$
\begin{aligned}
\left.\frac{1}{L^{9}}\left(a_{r}-\frac{\partial r_{c}}{\partial \theta} a_{\theta}\right)\right|_{r=r_{c}(\theta, \varepsilon)}= & \frac{5 \sin \theta \cos ^{2} \theta(\cos 2 \theta-5)\left(16+3 m_{2}^{2}-8 m_{3}\right)}{4 \varepsilon^{2}} \\
& +\frac{\sin 2 \theta}{128}\left[\left(2144 m_{2}+445 m_{2}^{3}-1760 m_{2} m_{3}-860 m_{4}\right.\right. \\
& +20 \cos 2 \theta\left(64 m_{2}+15 m_{2}^{3}-80 m_{2} m_{3}-60 m_{4}\right) \\
& \left.+\cos 4 \theta\left(-96 m_{2}-25 m_{2}^{3}+160 m_{2} m_{3}+140 m_{4}\right)\right] \\
& +O\left(\varepsilon^{2}\right)
\end{aligned}
$$

After performing the integration over $\theta$ the finite terms above drop out and and we are left with

$$
I_{(\text {cut })}=-\frac{L^{9}}{48 \pi G_{N}^{(11)}} \operatorname{Vol}\left(S^{3}\right)^{2} \operatorname{Vol}\left(A d S_{3}\right)\left[\frac{-64-12 m_{2}^{2}+32 m_{3}}{\varepsilon^{2}}+O\left(\varepsilon^{2}\right)\right]
$$

\subsubsection{Real line contribution}

The expression from the real line can be obtained from expanding the integrand of (5.29) in the $y \rightarrow 0$ limit. The behavior of the integrand in this limit depends on the interval $x$ is located in. We define

$$
\begin{aligned}
\mathcal{I}_{0} & =\left[-\infty, \xi_{1}\right] \cup\left[\xi_{2}, \xi_{3}\right] \cup \cdots \cup\left[\xi_{2 n},+\infty\right] \\
\mathcal{I}_{+} & =\left[\xi_{1}, \xi_{2}\right] \cup\left[\xi_{3}, \xi_{4}\right] \cup \cdots \cup\left[\xi_{2 n-1}, \xi_{2 n}\right]
\end{aligned}
$$

We present the details of the calculation in appendix $\mathrm{D}$, with the final result given by

$$
\lim _{y \rightarrow 0}\left(-\frac{f_{2}^{3} f_{3}^{3}}{2 f_{1}^{3}} \partial_{y}\left(b_{1}^{2}\right)+\frac{f_{1}^{3} f_{3}^{3}}{2 f_{2}^{3}} \partial_{y}\left(b_{2}^{2}\right)+\frac{f_{1}^{3} f_{2}^{3}}{2 f_{3}^{3}} \partial_{y}\left(b_{3}^{2}\right)\right)= \begin{cases}\frac{16 L^{9}\left(2 g_{1}^{3}+g_{3}\right) \phi_{0}}{\left(g_{1}^{2}-g_{2}\right)\left(g_{1}^{2}+g_{2}\right)} & x \in \mathcal{I}_{0} \\ \frac{32 L^{9}\left(g_{1}^{3}+3 g_{1} g_{2}-g_{3}\right)\left(\phi_{0}+2 x\right)}{\left(g_{1}^{2}+g_{2}\right)\left(g_{1}^{2}+2 g_{2}\right)} & x \in \mathcal{I}_{+}\end{cases}
$$


where the $g_{i}$ and $\phi_{0}$ are functions of $x$ and can be found in (D.3) and (D.5), respectively. In this limit the integrand (5.33) is nonsingular for any finite $x$ on the real line. However, the integrand grows as $x \rightarrow \pm \infty$ and we can extract the divergent behavior coming from the region near the cut-off. We determine the divergent contributions in (D.14) and we write the result as

$$
I_{(x)}=+\frac{L^{9}}{48 \pi G_{N}^{(11)}} \operatorname{Vol}\left(S^{3}\right)^{2} \operatorname{Vol}\left(A d S_{3}\right)\left[-\frac{256}{\varepsilon^{6}}+\frac{80-12 m_{2}^{2}+32 m_{3}}{\varepsilon^{2}}+\mathcal{F}+O\left(\varepsilon^{2}\right)\right]
$$

The finite term $\mathcal{F}$ can in principle be determined by performing the $x$-integral for the full integration region and subtracting the divergent contributions given above, i.e.

$$
\begin{aligned}
\mathcal{F} \equiv & \lim _{\varepsilon \rightarrow 0}\left\{\sum_{x \in \mathcal{I}_{+}} \int d x \frac{32\left(g_{1}^{3}+3 g_{1} g_{2}-g_{3}\right)\left(\phi_{0}+2 x\right)}{\left(g_{1}^{2}+g_{2}\right)\left(g_{1}^{2}+2 g_{2}\right)}+\sum_{x \in \mathcal{I}_{0}} \int d x \frac{16\left(2 g_{1}^{3}+g_{3}\right) \phi_{0}}{\left(g_{1}^{2}-g_{2}\right)\left(g_{1}^{2}+g_{2}\right)}\right. \\
& \left.-\left(-\frac{256}{\varepsilon^{6}}+\frac{80-12 m_{2}^{2}+32 m_{3}}{\varepsilon^{2}}\right)\right\}
\end{aligned}
$$

The last two terms remove the divergent contributions from the integral over $\mathcal{I}_{0}$ that is regulated for large positive $x$ by $x<x_{c,+}(\varepsilon)$ and for large negative $x$ by $x>x_{c,-}(\varepsilon)$.

At this point we have not been able to find a closed expression for these integrals, but they can be evaluated numerically. If a relation to a matrix model calculation exists these integrals may be related to the resolvent. However, as no proposal for a matrix model exists at present we have not pursued the evaluation further and leave this for future work.

\subsection{Final result}

Combining all the contributions to the action, which can be found in (5.23), (5.31) and (5.34), we obtain for the on-shell action

$$
\begin{aligned}
I & =\frac{L^{9}}{3 \pi G_{N}^{(11)}} \operatorname{Vol}\left(S^{3}\right)^{2} \operatorname{Vol}\left(A d S_{3}\right)\left[\frac{80}{\varepsilon^{6}}+\frac{3}{\varepsilon^{2}}+\frac{\mathcal{F}}{16}+O\left(\varepsilon^{2}\right)\right] \\
& =\frac{N^{3}}{12 \pi} \operatorname{Vol}\left(A d S_{3}\right)\left(\frac{80}{\varepsilon^{6}}+\frac{3}{\varepsilon^{2}}+\frac{\mathcal{F}}{16}+O\left(\varepsilon^{2}\right)\right)
\end{aligned}
$$

The finite contribution is given by (5.35). This can be evaluated exactly for the vacuum and the result is

$$
\mathcal{F}_{(0)}=-64
$$

Thus, using (5.1) we can express our final result for the expectation value of the Wilson surface operator as

$$
\log \left\langle W_{\Gamma}\right\rangle=-\frac{N^{3}}{192 \pi} \operatorname{Vol}\left(A d S_{3}\right)(\mathcal{F}+64)
$$

Note that the power divergences in $\varepsilon$ are independent of the details of the bubbling geometry and so cancel in the subtraction. However, the result is proportional to the infinite volume 
of $A d S_{3}$, which is regulated by the cut-off $z=\eta$ introduced in the entanglement entropy calculation in (3.13).

As is the case for the stress tensor contribution, the expectation value of the Wilson surface operator constitutes a potentially useful holographic observable of the $(2,0)$ theory. This holographic result may be compared to direct calculations in this theory as well as be applied to the replica calculation of the entanglement entropy. It would be very interesting to study localization and related methods to calculate the expectation value of Wilson surface operators in the future.

\section{Discussion}

In this paper we have calculated the holographic entanglement entropy for the sixdimensional $(2,0)$ theory in the presence of a Wilson surface. In addition we have calculated two other holographic observables: the one point function of the stress tensor in the presence of a Wilson surface and the expectation value of the Wilson surface operator. The bubbling solution that provides the holographic dual of the 'heavy' Wilson surface is determined in terms of $2 n$ real numbers $\xi_{i}$ that parametrize the partition of the real line into two types of segment (where $G=0$ or $G=+i$ ). The quantities we calculated all depend on moments $m_{k}$ whose definition in terms of $\xi_{i}$ is given in (2.18). In particular, the stress tensor only depends on $m_{2}$ and $m_{3}$. On the other hand, the final term in the result for the entanglement entropy (3.18) cannot in general be expressed in terms of the moments $m_{k}$. The finite part of the expectation value of the Wilson surface operator is expressed in terms of a real integral. While it is possible to evaluate this finite part for $n=1$ and $n=2$ in closed form, we have been unable to evaluate it in general. Some numerical experiments however indicate that this integral does not have a simple expression in terms of the moments $m_{k}$ or the final term in the entanglement entropy (3.18).

This 'coloring' of the real line is reminiscent of other bubbling solutions, such as those dual to Wilson loops [14] or the LLM solutions [46], where the data of the solution can be mapped to the data of a matrix model or free fermion phase space, respectively. One hint that BPS Wilson surfaces might be described by matrix models can be seen as follows. If one compactifies the six-dimensional $(2,0)$ theory on a circle one obtains five-dimensional SYM theory $[47,48]$ and a Wilson surface that wraps the circle becomes a Wilson line in this five-dimensional gauge theory [32, 49]. Various quantities have been calculated for this system using localization and it would be very interesting to see if these results can be lifted to six dimensions and compared to our calculations in the limit of very large 'representations', in analogy with [21].

It would also be interesting to see whether the calculation of the entanglement entropy in the presence of a Wilson loop in $\mathrm{SU}(N) \mathcal{N}=4 \mathrm{SYM}$ due to Lewkowycz and Maldacena [17] generalizes to the Wilson surface in the $(2,0)$ theory. Recall that their calculation used the replica trick and involved the expectation value of the Wilson loop and the stress tensor in the presence of the Wilson loop on the space $S^{1} \times H^{3}$. A generalization would most likely start from the expectation value the Wilson surface and the stress tensor in the presence of the Wilson surface on $S^{1} \times H^{5}$. Since our holographic calculation gives these two quantities for the $A d S_{3} \times S^{3}$ boundary, if it is possible to map the results to $S^{1} \times H^{5}$ 
then it should be possible to compare the Lewkowycz and Maldacena calculation to the holographic entanglement entropy calculation we have performed. One complication is that unlike a one-dimensional Wilson loop, the two-dimensional Wilson surface has a conformal anomaly [50-52] and it is not clear how to determine its contribution in our case. A simpler case in which to consider the anomaly might be the case of an abelian Wilson surface, as studied in [52].

Our calculations provide results that could be compared to any field theory or localization calculation in the $(2,0)$ theory. What is missing at this point, compared to the analogous case of the half-BPS Wilson loop in $\mathcal{N}=4 \mathrm{SYM}$, is a concrete dictionary between a matrix model and the supergravity parameters as well as a better understanding of the anomaly and the map of the stress tensor and the expectation value to the $S^{1} \times H^{5}$ boundary geometry. We leave these interesting questions for future work.

\section{Acknowledgments}

It is a pleasure to thank Eric D'Hoker, Per Kraus and Christoph Uhlemann for useful discussions. This work was supported in part by National Science Foundation grant PHY13-13986.

\section{A Contributions to the entanglement entropy}

In this appendix we carefully discuss the contribution to the area integrals that are needed in section 3.2.

\section{A.1 $J_{1}$}

First we consider $J_{1}$, given in (3.9). The radial integral can be performed directly, but it is useful to rewrite it in terms of Legendre polynomials using (2.15). We divide the integration range into two regions: $0 \leq r \leq\left|\xi_{i}\right|$ and $\left|\xi_{i}\right| \leq r \leq r_{c}(\theta, \varepsilon)$. For each region we choose the Legendre representation that converges, yielding

$$
\begin{aligned}
J_{1}= & -4 L^{9} \sum_{i=1}^{2 n}(-1)^{i} \int_{0}^{\pi} d \theta \sin \theta\left\{\int_{\left|\xi_{i}\right|}^{r_{c}(\theta, \varepsilon)} d r \frac{r^{2}\left(r \cos \theta-\xi_{i}\right)}{r} \sum_{\ell=0}^{\infty} P_{\ell}(\cos \theta)\left(\frac{\xi_{i}}{r}\right)^{\ell}\right. \\
& \left.+\int_{0}^{\left|\xi_{i}\right|} d r \frac{r^{2}\left(r \cos \theta-\xi_{i}\right)}{\left|\xi_{i}\right|} \sum_{\ell=0}^{\infty} P_{\ell}(\cos \theta)\left(\frac{r}{\xi_{i}}\right)^{\ell}\right\}
\end{aligned}
$$

Performing the two radial integrals directly we find

$$
\begin{aligned}
J_{1}= & -4 L^{9} \sum_{i=1}^{2 n}(-1)^{i} \int_{0}^{\pi} d \theta \sin \theta\left\{\left[\cos \theta P_{0} \frac{r^{3}}{3}+\left(\cos \theta P_{1}-P_{0}\right) \xi_{i} \frac{r^{2}}{2}+\left(\cos \theta P_{2}-P_{1}\right) \xi_{i}^{2} r\right.\right. \\
& \left.+\left(\cos \theta P_{3}-P_{2}\right) \xi_{i}^{3} \log r-\sum_{\ell=1}^{\infty} \frac{\left(\cos \theta P_{\ell+3}-P_{\ell+2}\right)}{\ell} \frac{\xi_{i}^{\ell+3}}{r^{\ell}}\right]_{\left|\xi_{i}\right|}^{r_{c}(\theta, \varepsilon)} \\
& \left.+\frac{1}{\left|\xi_{i}\right|}\left[-P_{0} \xi_{i} \frac{r^{3}}{3}+\sum_{\ell=4}^{\infty} \frac{\left(\cos \theta P_{\ell-4}-P_{\ell-3}\right)}{\ell} \frac{r^{\ell}}{\xi_{i}^{\ell-4}}\right]_{0}^{\left|\xi_{i}\right|}\right\}
\end{aligned}
$$


Orthogonality of the Legendre polynomials can be expressed via

$$
\int_{0}^{\pi} d \theta \sin \theta P_{\ell}(\cos \theta) P_{k}(\cos \theta)=\frac{2}{2 \ell+1} \delta_{\ell k}
$$

We use this to simplify the above expression dramatically:

$$
\begin{aligned}
J_{1}= & -4 L^{9} \sum_{i=1}^{2 n}(-1)^{i}\left\{\int _ { 0 } ^ { \pi } d \theta \operatorname { s i n } \theta \left[\cos \theta P_{0} \frac{r_{c}^{3}}{3}+\left(\cos \theta P_{1}-P_{0}\right) \xi_{i} \frac{r_{c}^{2}}{2}+\left(\cos \theta P_{2}-P_{1}\right) \xi_{i}^{2} r_{c}\right.\right. \\
& \left.\left.+\left(\cos \theta P_{3}-P_{2}\right) \xi_{i}^{3} \log r_{c}-\sum_{\ell=1}^{\infty} \frac{\left(\cos \theta P_{\ell+3}-P_{\ell+2}\right)}{\ell} \frac{\xi_{i}^{\ell+3}}{r_{c}^{\ell}}\right]+\frac{2}{15} \xi_{i}^{3}\right\}
\end{aligned}
$$

Note that the final term is a sum of contributions at $r=\left|\xi_{i}\right|$. Substituting for the cutoff function $r_{c}(\theta, \varepsilon)$ given in (2.26), we then expand in $\varepsilon$ up to and including $O\left(\varepsilon^{0}\right)$ and perform the remaining integrals over $\theta$. We find the final result

$$
J_{1}=L^{9}\left[\frac{64}{3 \varepsilon^{4}}+\frac{-24+3 m_{2}^{2}-8 m_{3}}{15}+O\left(\varepsilon^{2}\right)\right]
$$

\section{A.2 $J_{2}$}

Next we calculate $J_{2}$, which we reproduce from (3.11):

$$
\begin{aligned}
J_{2}= & -2 L^{9} \int_{0}^{\pi} d \theta \sin \theta \int_{0}^{r_{c}(\theta, \varepsilon)} d r r^{2} \\
& \times\left\{2 n+2 \sum_{i<j}(-1)^{i+j} \frac{r^{2}-r \cos \theta\left(\xi_{i}+\xi_{j}\right)+\xi_{i} \xi_{j}}{\sqrt{r^{2}-2 r \xi_{i} \cos \theta+\xi_{i}^{2}} \sqrt{r^{2}-2 r \xi_{j} \cos \theta+\xi_{j}^{2}}}\right\}
\end{aligned}
$$

We can split the integral into two terms coming from the sum in the last line of (A.6). The first term is simply

$$
\begin{aligned}
J_{2, a} & \equiv-\frac{4}{3} n L^{9} \int_{0}^{\pi} d \theta \sin \theta r_{c}(\theta, \varepsilon)^{3} \\
& =L^{9}\left[-\frac{64 n}{3 \varepsilon^{6}}+\frac{n\left(40+3 m_{2}^{2}-8 m_{3}\right)}{18 \varepsilon^{2}}+O\left(\varepsilon^{2}\right)\right]
\end{aligned}
$$

The evaluation of the second term, denoted $J_{2, b} \equiv J_{2}-J_{2, a}$, is more involved than that of $J_{1}$. Our strategy is to divide up the radial integration range and replace the square root factors with the appropriate convergent series of Legendre polynomials in each interval. The fraction in the summand is symmetric under $(i \leftrightarrow j)$ so we can choose $\left|\xi_{i}\right|<\left|\xi_{j}\right|$ 
without loss of generality and write:

$$
\begin{aligned}
J_{2, b}= & -4 L^{9} \sum_{i<j}(-1)^{i+j} \int_{0}^{\pi} d \theta \sin \theta \\
& \times\left\{\int_{\left|\xi_{j}\right|}^{r_{c}(\theta, \varepsilon)} d r \frac{r^{2}\left(r^{2}-r \cos \theta\left(\xi_{i}+\xi_{j}\right)+\xi_{i} \xi_{j}\right)}{r^{2}} \sum_{\ell=0}^{\infty} P_{\ell}\left(\frac{\xi_{i}}{r}\right)^{\ell} \sum_{k=0}^{\infty} P_{k}\left(\frac{\xi_{j}}{r}\right)^{k}\right. \\
& +\int_{\left|\xi_{i}\right|}^{\left|\xi_{j}\right|} d r \frac{r^{2}\left(r^{2}-r \cos \theta\left(\xi_{i}+\xi_{j}\right)+\xi_{i} \xi_{j}\right)}{r\left|\xi_{j}\right|} \sum_{\ell=0}^{\infty} P_{\ell}\left(\frac{\xi_{i}}{r}\right)^{\ell} \sum_{k=0}^{\infty} P_{k}\left(\frac{r}{\xi_{j}}\right)^{k} \\
& \left.+\int_{0}^{\left|\xi_{i}\right|} d r \frac{r^{2}\left(r^{2}-r \cos \theta\left(\xi_{i}+\xi_{j}\right)+\xi_{i} \xi_{j}\right)}{\left|\xi_{i}\right|\left|\xi_{j}\right|} \sum_{\ell=0}^{\infty} P_{\ell}\left(\frac{r}{\xi_{i}}\right)^{\ell} \sum_{k=0}^{\infty} P_{k}\left(\frac{r}{\xi_{j}}\right)^{k}\right\} \\
\equiv & K_{1}+K_{2}+K_{3}
\end{aligned}
$$

where the Legendre polynomials are all functions of $\cos \theta$, as before. First let us consider $K_{1}$ :

$$
\begin{aligned}
K_{1} \equiv & -4 L^{9} \sum_{i<j}(-1)^{i+j} \int_{0}^{\pi} d \theta \sin \theta \\
& \times \sum_{\ell, k=0}^{\infty} P_{\ell} P_{k} \xi_{i}^{\ell} \xi_{j}^{k} \int_{\left|\xi_{j}\right|}^{r_{c}(\theta, \varepsilon)} d r\left(r^{2}-r \cos \theta\left(\xi_{i}+\xi_{j}\right)+\xi_{i} \xi_{j}\right) r^{-\ell-k}
\end{aligned}
$$

We must perform the radial integral first because its upper limit depends on $\theta$. This results in several sums over powers of $r$ and a logarithm:

$$
\left.\left.\begin{array}{rl}
K_{1}= & -4 L^{9} \sum_{i<j}(-1)^{i+j} \int_{0}^{\pi} d \theta \sin \theta\left\{\sum_{\substack{\ell, k=0 \\
\ell+k \neq 3}}^{\infty} \frac{P_{\ell} P_{k} \xi_{i}^{\ell} \xi_{j}^{k} r^{3-\ell-k}}{3-\ell-k}\right. \\
& -\cos \theta\left(\xi_{i}+\xi_{j}\right) \sum_{\substack{\ell, k=0 \\
\ell+k \neq 2}}^{\infty} \frac{P_{\ell} P_{k} \xi_{i}^{\ell} \xi_{j}^{k} r^{2-\ell-k}}{2-\ell-k}+\xi_{i} \xi_{j} \sum_{\substack{\ell, k=0 \\
\ell+k \neq 1}}^{\infty} \frac{P_{\ell} P_{k} \xi_{i}^{\ell} \xi_{j}^{k} r^{1-\ell-k}}{1-\ell-k} \\
& +\left(\sum_{\substack{\ell, k=0 \\
\ell+k=3}}^{3} P_{\ell} P_{k} \xi_{i}^{\ell} \xi_{j}^{k}-\cos \theta\left(\xi_{i}+\xi_{j}\right) \sum_{\substack{\ell, k=0 \\
\ell+k=2}}^{2} P_{\ell} P_{k} \xi_{i}^{\ell} \xi_{j}^{k}+\xi_{i} \xi_{j} \sum_{\substack{\ell, k=0 \\
\ell+k=1}}^{1} P_{\ell} P_{k} \xi_{i}^{\ell} \xi_{j}^{k}\right.
\end{array}\right) \log r\right\}_{\left|\xi_{j}\right|}^{r_{c}(\theta, \varepsilon)}
$$

We only require the entanglement entropy up to and including $O\left(\varepsilon^{0}\right)$. Recall that the cut-off function $r_{c}(\theta, \varepsilon)$ given (2.26) leads with $O\left(\varepsilon^{-2}\right)$, and therefore only the logarithm and non-negative powers of $r$ contribute to the upper limit. Specifically, we can terminate the infinite sums in the first and second lines at 3,2 and 1 , respectively. Integrating over $\theta$ we find

$$
K_{1}^{\text {upper }}=L^{9}\left[\frac{64 n}{3 \varepsilon^{6}}-\frac{n\left(40+3 m_{2}^{2}-8 m_{3}\right)}{18 \varepsilon^{2}}-\frac{64}{3 \varepsilon^{2}}+O\left(\varepsilon^{2}\right)\right]
$$


where we have made use of the following results:

$$
\sum_{i<j}(-1)^{i+j}=-n, \quad \sum_{i<j}(-1)^{i+j}\left(\xi_{i}-\xi_{j}\right)^{2}=-4
$$

Next let us consider the lower limit and perform the integral over $\theta$. The terms with no explicit $\cos \theta$ factor vanish unless $\ell=k$ by orthogonality (A.3). To deal with the terms that do have an explicit $\cos \theta$ factor, let us define

$$
\begin{aligned}
X_{\ell k} & \equiv \int_{0}^{\pi} d \theta \sin \theta \cos \theta P_{\ell}(\cos \theta) P_{k}(\cos \theta)=2\left(\begin{array}{lll}
1 & \ell & k \\
0 & 0 & 0
\end{array}\right)^{2} \\
& =\frac{2(\ell-k)^{2}(1+\ell+k)}{(\ell+k)(2+\ell+k)(1+\ell-k) !(1-\ell+k) !}
\end{aligned}
$$

These terms are only non-zero when $X_{\ell k}$ is too, which occurs when $|\ell-k|=1$. These two observations imply that the coefficient of the logarithm vanishes and that the conditions on the sums in the first two lines of (A.10) have no effect for the lower limit. All that remains is

$$
\begin{aligned}
K_{1}^{\text {lower }}= & +4 L^{9} \sum_{i<j}(-1)^{i+j} \sum_{\ell, k=0}^{\infty} \xi_{i}^{\ell} \xi_{j}^{k}\left[\frac{2}{2 \ell+1} \delta_{\ell k}\left(\frac{\left|\xi_{j}\right|^{3-\ell-k}}{3-\ell-k}+\frac{\left|\xi_{j}\right|^{1-\ell-k}}{1-\ell-k} \xi_{i} \xi_{j}\right)\right. \\
& \left.-\frac{\left|\xi_{j}\right|^{2-\ell-k}}{2-\ell-k}\left(\xi_{i}+\xi_{j}\right) X_{\ell k}\right]
\end{aligned}
$$

Performing the sum over $k$ and using the definition (A.13) we find

$$
\begin{aligned}
K_{1}^{\text {lower }}= & +4 L^{9} \sum_{i<j}(-1)^{i+j} \sum_{\ell=0}^{\infty} \frac{2}{2 \ell+1} \xi_{i}^{\ell} \xi_{j}^{\ell}\left[\frac{\left|\xi_{j}\right|^{3-2 \ell}}{3-2 \ell}+\frac{\left|\xi_{j}\right|^{1-2 \ell}}{1-2 \ell} \xi_{i} \xi_{j}\right. \\
& \left.-\frac{(\ell+1)\left|\xi_{j}\right|^{1-2 \ell}}{(2 \ell+3)(1-2 \ell)}\left(\xi_{i}+\xi_{j}\right)^{2}\right]
\end{aligned}
$$

The limits on the radial integrals in $K_{2,3}$ are independent of $\theta$ so we are free to reverse the order of integration. Let us begin with $K_{2}$ :

$$
\begin{aligned}
K_{2} \equiv & -4 L^{9} \sum_{i<j} \frac{(-1)^{i+j}}{\left|\xi_{j}\right|} \int_{\left|\xi_{i}\right|}^{\left|\xi_{j}\right|} d r \sum_{\ell, k=0}^{\infty} r^{k-\ell} \frac{\xi_{i}^{\ell}}{\xi_{j}^{k}} \\
& \times \int_{0}^{\pi} d \theta \sin \theta P_{\ell} P_{k}\left(r^{3}-r^{2} \cos \theta\left(\xi_{i}+\xi_{j}\right)+r \xi_{i} \xi_{j}\right) \\
= & -4 L^{9} \sum_{i<j} \frac{(-1)^{i+j}}{\left|\xi_{j}\right|} \int_{\left|\xi_{i}\right|}^{\left|\xi_{j}\right|} d r \sum_{\ell, k=0}^{\infty} r^{k-\ell} \frac{\xi_{i}^{\ell}}{\xi_{j}^{k}}\left[\frac{2}{2 \ell+1} \delta_{\ell k}\left(r^{3}+r \xi_{i} \xi_{j}\right)-r^{2}\left(\xi_{i}+\xi_{j}\right) X_{\ell k}\right]
\end{aligned}
$$

where again we have used (A.3) and (A.13). Performing the sum over $k$ then integrating over $r$ we find

$$
\begin{aligned}
K_{2}= & -4 L^{9} \sum_{i<j} \frac{(-1)^{i+j}}{\left|\xi_{j}\right|} \sum_{\ell=0}^{\infty} \frac{2}{2 \ell+1} \frac{\xi_{i}^{\ell}}{\xi_{j}^{\ell}}\left[1-\frac{\ell+1}{2 \ell+3}\left(1+\frac{\xi_{i}}{\xi_{j}}\right)\right] \\
& \times\left(\frac{\left|\xi_{j}\right|^{4}-\left|\xi_{i}\right|^{4}}{4}+\frac{\left|\xi_{j}\right|^{2}-\left|\xi_{i}\right|^{2}}{2} \xi_{i} \xi_{j}\right)
\end{aligned}
$$


We can compute $K_{3}$ using the same method:

$$
\begin{aligned}
K_{3} \equiv & -4 L^{9} \sum_{i<j} \frac{(-1)^{i+j}}{\left|\xi_{i}\right|\left|\xi_{j}\right|} \int_{0}^{\left|\xi_{i}\right|} d r \sum_{\ell, k=0}^{\infty} \frac{r^{\ell+k}}{\xi_{i}^{\ell \xi_{j}^{k}}} \\
& \times \int_{0}^{\pi} d \theta \sin \theta P_{\ell} P_{k}\left(r^{4}-r^{3} \cos \theta\left(\xi_{i}+\xi_{j}\right)+r^{2} \xi_{i} \xi_{j}\right) \\
= & -4 L^{9} \sum_{i<j} \frac{(-1)^{i+j}}{\left|\xi_{i}\right|\left|\xi_{j}\right|} \int_{0}^{\left|\xi_{i}\right|} d r \sum_{\ell, k=0}^{\infty} \frac{r^{\ell+k}}{\xi_{i}^{\ell} \xi_{j}^{k}}\left[\frac{2}{2 \ell+1} \delta_{\ell k}\left(r^{4}+r^{2} \xi_{i} \xi_{j}\right)-r^{3}\left(\xi_{i}+\xi_{j}\right) X_{\ell k}\right] \\
= & -4 L^{9} \sum_{i<j} \frac{(-1)^{i+j}}{\left|\xi_{i}\right|\left|\xi_{j}\right|} \sum_{\ell=0}^{\infty} \frac{2}{2 \ell+1} \frac{1}{\xi_{i}^{\ell} \xi_{j}^{\ell}}\left\{\left[1-\frac{\ell+1}{2 \ell+3}\left(\xi_{i}+\xi_{j}\right)\left(\frac{1}{\xi_{i}}+\frac{1}{\xi_{j}}\right)\right] \frac{\left|\xi_{i}\right|^{5+2 \ell}}{5-2 \ell}\right. \\
& \left.+\frac{\left|\xi_{i}\right|^{3+3 \ell}}{3+2 \ell} \xi_{i} \xi_{j}\right\}
\end{aligned}
$$

Now we combine the finite contributions to $J_{2, b}$. The infinite sums can be evaluated and the following remarkably simple result is obtained:

$$
K_{1}^{\text {lower }}+K_{2}+K_{3}=\frac{4 L^{9}}{3} \sum_{i<j}(-1)^{i+j} \operatorname{sgn} \xi_{j}\left(\xi_{i}-\xi_{j}\right)^{3}
$$

Recall that we have assumed $\left|\xi_{i}\right|<\left|\xi_{j}\right|$. The ordering of the $\xi_{i}$ in (2.13) implies that $\operatorname{sgn} \xi_{j}$ evaluates to +1 and since the sum is ordered we can write the finite contribution to $J_{2, b}$ as

$$
-\frac{4 L^{9}}{3} \sum_{i<j}(-1)^{i+j}\left|\xi_{i}-\xi_{j}\right|^{3}
$$

Note that this term cannot be expressed in terms of the moments $m_{k}$. Thus, our final result for $J_{2, b}$ is:

$$
J_{2, b}=L^{9}\left[\frac{64 n}{3 \varepsilon^{6}}-\frac{n\left(40+3 m_{2}^{2}-8 m_{3}\right)}{18 \varepsilon^{2}}-\frac{64}{3 \varepsilon^{2}}-\frac{4}{3} \sum_{i<j}(-1)^{i+j}\left|\xi_{i}-\xi_{j}\right|^{3}+O\left(\varepsilon^{2}\right)\right]
$$

Summing (A.7) and (A.21) we find

$$
J_{2}=L^{9}\left[-\frac{64}{3 \varepsilon^{2}}-\frac{4}{3} \sum_{i<j}(-1)^{i+j}\left|\xi_{i}-\xi_{j}\right|^{3}+O\left(\varepsilon^{2}\right)\right]
$$

\section{B Calculation of the holographic stress tensor}

In this appendix we present some details of the KK reduction calculation as well as the calculation of the stress tensor using holographic renormalization. As mentioned in section 4, first one has to decompose the metric into the vacuum $A d S_{7} \times S^{4}$ part and fluctuations, as in (4.2). In FG coordinates the vacuum metric is given by

$$
\begin{aligned}
g_{M N}^{(0)} d x^{M} d x^{N}= & L^{2}\left[\frac{4}{u^{2}}\left(d u^{2}+\left(1+\frac{u^{2}}{2}+\frac{u^{4}}{16}\right) d s_{A d S_{3}}^{2}+\left(1-\frac{u^{2}}{2}+\frac{u^{4}}{16}\right) d s_{S^{3}}^{2}\right)\right. \\
& \left.+d \tilde{\theta}^{2}+\sin ^{2} \tilde{\theta} d s_{\tilde{S}_{3}}^{2}\right] .
\end{aligned}
$$


The metric fluctuations in terms of the functions $\alpha_{i}(u, \tilde{\theta})$ appearing in $(2.21)$ are

$$
\begin{aligned}
h_{M N} d x^{M} d x^{N}= & L^{2}\left[\frac{4}{u^{2}}\left\{\left(\alpha_{1}-1-\frac{u^{2}}{2}-\frac{u^{4}}{16}\right) d s_{A d S_{3}}^{2}+\left(\alpha_{2}-1+\frac{u^{2}}{2}-\frac{u^{4}}{16}\right) d s_{S^{3}}^{2}\right\}\right. \\
& \left.+\left(\alpha_{3}-1\right) d \tilde{\theta}^{2}+\left(\alpha_{4}-\sin ^{2} \tilde{\theta}\right) d s_{\tilde{S}_{3}}^{2}\right] .
\end{aligned}
$$

Using these expressions, we calculate the seven-dimensional reduced metric (4.4) and the outcome is

$$
\begin{aligned}
d s_{7}^{2}= & \frac{4 L^{2}}{u^{2}}\left[d u^{2}+\left(1+\frac{u^{2}}{2}+\frac{u^{4}}{16}+\frac{1}{320}\left(16+3 m_{2}^{2}-8 m_{3}\right) u^{6}\right) d s_{A d S_{3}}^{2}\right. \\
& \left.+\left(1-\frac{u^{2}}{2}+\frac{u^{4}}{16}-\frac{1}{320}\left(16+3 m_{2}^{2}-8 m_{3}\right) u^{6}\right) d s_{S^{3}}^{2}\right]
\end{aligned}
$$

Notice that substituting the vacuum moments in (B.3) one can retrieve the $A d S_{7}$ entries in (B.1). This is because the trace shift does not contribute to the reduced metric, i.e. $\bar{\pi}$ vanishes. Furthermore, a further FG map of (B.3) is not necessary since it is already in FG form:

$$
d s_{7}^{2}=\frac{4 L^{2}}{u^{2}}\left(d u^{2}+g_{i j} d x^{i} d x^{j}\right)
$$

where the six-dimensional metric $g_{i j}$ is given by a power series in $u$ :

$$
g=g_{(0)}+g_{(2)} u^{2}+g_{(4)} u^{4}+g_{(6)} u^{6}+h_{(6)} u^{6} \log u^{2}+\ldots
$$

To compute the holographic stress tensor, we simply read off the asymptotic metric coefficients $g_{(0)}, g_{(2)}, g_{(4)}$ and $g_{(6)}$ from (B.3) and substitute them into the $d=6$ formula given in [44]. For completeness we present this fomula here:

$$
\left\langle T_{i j}\right\rangle=\frac{3(2 L)^{5}}{8 \pi G_{N}^{(7)}}\left(g_{(6) i j}-A_{(6) i j}+\frac{1}{24} S_{i j}\right)
$$

where the second and third terms are defined via

$$
\begin{aligned}
A_{(6) i j}= & \frac{1}{3}\left[2\left(g_{(2)} g_{(4)}\right)_{i j}+\left(g_{(4)} g_{(2)}\right)_{i j}-\left(g_{(3)}^{3}\right)_{i j}+\frac{1}{8} g_{(2) i j}\left(\operatorname{tr} g_{(2)}^{2}-\left(\operatorname{tr} g_{(2)}\right)^{2}\right)\right. \\
& -\operatorname{tr} g_{(2)}\left(g_{(4) i j}-\frac{1}{2}\left(g_{(2)}^{2}\right)_{i j}\right)-g_{(0) i j}\left(\frac{1}{8} \operatorname{tr} g_{(2)}^{2} \operatorname{tr} g_{(2)}-\frac{1}{24}\left(\operatorname{tr} g_{(2)}\right)^{3}\right. \\
& \left.\left.-\frac{1}{6} \operatorname{tr} g_{(2)}^{3}+\frac{1}{2} \operatorname{tr}\left(g_{(2)} g_{(4)}\right)\right)\right] \\
S_{i j}= & \nabla^{2} C_{i j}-2 R_{i j}^{k} C_{k l}+4\left(\left(g_{(2)} g_{(4)}\right)-\left(g_{(4)} g_{(2)}\right)\right)_{i j}+\frac{1}{10}\left(\nabla_{i} \nabla_{j} B-g_{(0) i j} \nabla^{2} B\right) \\
& +\frac{2}{5} g_{(2) i j} B+g_{(0) i j}\left(-\frac{2}{3} \operatorname{tr} g_{(2)}^{3}-\frac{4}{15}\left(\operatorname{tr} g_{(2)}\right)^{3}+\frac{3}{5} \operatorname{tr} g_{(2)} \operatorname{tr} g_{(2)}^{2}\right)
\end{aligned}
$$

with the quantities $C_{i j}$ and $B$ defined by

$$
\begin{aligned}
C_{i j} & =g_{(4) i j}-\frac{1}{2}\left(g_{(2)}^{2}\right)_{i j}+\frac{1}{4} g_{(2) i j} \operatorname{tr} g_{(2)}+\frac{1}{8} g_{(0) i j} B \\
B & =\operatorname{tr} g_{(2)}^{2}-\left(\operatorname{tr} g_{(2)}\right)^{2}
\end{aligned}
$$


Note that the contraction of indices is performed with the inverse of $g_{(0) i j}$. A general formula for the trace of the stress tensor follows from these definitions:

$$
\left\langle T_{i}^{i}\right\rangle=\frac{3(2 L)^{5}}{8 \pi G_{N}^{(7)}}\left(-\frac{1}{24}\left(\operatorname{tr} g_{(2)}\right)^{3}+\frac{1}{8} \operatorname{tr} g_{(2)} \operatorname{tr} g_{(2)}^{2}-\frac{1}{6} \operatorname{tr} g_{(2)}^{3}+\frac{1}{3} \operatorname{tr} g_{(2)} g_{(4)}\right)
$$

Evaluating these formulae we find

$$
\left\langle T_{i j}\right\rangle d x^{i} d x^{j}=\frac{2^{4} L^{5}}{8 \pi G_{N}^{(7)}} \frac{20+9 m_{2}^{2}-24 m_{3}}{160}\left(d s_{A d S_{3}}^{2}-d s_{S^{3}}^{2}\right)
$$

Notice that the stress tensor is traceless, which reflects the fact there is no Weyl anomaly for $A d S_{3} \times S^{3}$. After observing

$$
\frac{1}{8 \pi G_{N}^{(7)}}=\frac{\operatorname{Vol}\left(S_{L}^{4}\right)}{8 \pi G_{N}^{(11)}}, \quad \operatorname{Vol}\left(S_{L}^{4}\right)=\frac{8 \pi^{2}}{3} L^{4}
$$

and using the definitions (3.15), we then subtract off the contribution from the vacuum to obtain our final result:

$$
\Delta\left\langle T_{i j}\right\rangle d x^{i} d x^{j}=\frac{N^{3}}{160 \pi^{3}}\left(16+3 m_{2}^{2}-8 m_{3}\right)\left(d s_{A d S_{3}}^{2}-d s_{S^{3}}^{2}\right)
$$

\section{Four-form field strength}

In this appendix we present the formula for the four-form field strength

$$
F=\left(f_{1}\right)^{3} g_{1 m} \omega_{A d S_{3}} \wedge e^{m}+\left(f_{2}\right)^{3} g_{2 m} \omega_{S^{3}} \wedge e^{m}+\left(f_{3}\right)^{3} g_{3 m} \omega_{\tilde{S}^{3}} \wedge e^{m}
$$

where $\omega_{X}$ denotes the volume form for a unit-radius space $X$. The $g_{I m}$ are related to derivatives of potentials $b_{I}$ via

$$
\begin{aligned}
& \left(f_{1}\right)^{3} g_{1 w}=-\partial_{w} b_{1} / L^{3}=2\left(j_{w}^{+}+j_{w}^{-}\right) \\
& \left(f_{2}\right)^{3} g_{2 w}=-\partial_{w} b_{2} / L^{3}=-2\left(j_{w}^{+}-j_{w}^{-}\right) \\
& \left(f_{3}\right)^{3} g_{3 w}=-\partial_{w} b_{3} / L^{3}=\frac{1}{8} j_{w}^{3}
\end{aligned}
$$

Since the four-form field strength is related to the three-form potentials by $F_{(I)}=d C_{(I)}$, it follows from (C.2) that the potentials take the following form:

$$
\begin{aligned}
& C_{(1)}=b_{1} \frac{1}{z^{3}} d z \wedge d t \wedge d l \\
& C_{(2)}=b_{2} \sin ^{2} \theta_{1} \sin \theta_{2} d \theta_{1} \wedge \theta_{2} \wedge d \theta_{3} \\
& C_{(3)}=b_{3} \sin ^{2} \psi_{1} \sin \psi_{2} d \psi_{1} \wedge d \psi_{2} \wedge d \psi_{3}
\end{aligned}
$$

Next we review the the expressions for the fields $j$ in (C.2) found in [37]. The currents can be expressed in a compact way by defining

$$
J_{w}=\frac{h}{L^{3}(G+\bar{G})}\left[\bar{G}\left(G-3 \bar{G}+4 G \bar{G}^{2}\right) \partial_{w} G+G(G+\bar{G}) \partial_{w} \bar{G}\right]
$$


and are given by

$$
\begin{aligned}
j_{w}^{+} & =2 i J_{w}\left((G-\bar{G})^{2}-4 G^{3} \bar{G}\right) W^{-4} \\
j_{w}^{-} & =2 G J_{w}\left(-2 G \bar{G}+3 \bar{G}^{2}-G^{2}+4 G^{2} \bar{G}^{2}\right) W^{-4} \\
j_{w}^{3} & =3 \partial_{w} h \frac{W^{2}}{G(1-G \bar{G})}-2 J_{w} \frac{\left(1+G^{2}\right)}{G(1-G \bar{G})^{2}}
\end{aligned}
$$

It is then straightforward to verify that the potentials are given by

$$
\begin{aligned}
& b_{1}=\frac{2(G+\bar{G}) h}{2 G \bar{G}+i(G-\bar{G})}+2 \tilde{h}-2 \Phi \\
& b_{2}=-\frac{2(G+\bar{G}) h}{2 G \bar{G}-i(G-\bar{G})}+2 \tilde{h}+2 \Phi \\
& b_{3}=-\frac{(G+\bar{G}) h}{4(G \bar{G}-1)}-\Phi
\end{aligned}
$$

Here, $\tilde{h}$ is the dual harmonic function to $h$ and satisfies

$$
i \partial_{w} h=\partial_{w} \tilde{h}
$$

With $h=-i L^{3}(w-\bar{w})$ as in (2.11), one obtains

$$
\tilde{h}=L^{3}(w+\bar{w})
$$

Also, $\Phi$ is defined via

$$
\bar{G} \partial_{w} h=\partial_{w} \Phi
$$

Using $\partial_{w} h=-i L^{3}$ and $G$ given by (2.12) we solve (C.9) to find

$$
\Phi=L^{3} \sum_{j}(-1)^{j} \sqrt{\left(w-\xi_{j}\right)\left(\bar{w}-\xi_{j}\right)}
$$

Note that $\Phi$ is real, hence the only thing that could be added is a constant, corresponding to an ambiguity in the definition of the $b_{I}$.

\section{Calculation of the real line contribution to the on-shell action}

In this appendix we present details on the calculation of the contribution from the real line to the on-shell action. To do this we have to expand the metric factors and $b_{I}$ in a power series in $y$ around $y=0$. The important point is that the expansion of $G, \bar{G}$ differs in different intervals. Let us define

$$
\begin{aligned}
\mathcal{I}_{0} & =\left[-\infty, \xi_{1}\right] \cup\left[\xi_{2}, \xi_{3}\right] \cup \cdots \cup\left[\xi_{2 n},+\infty\right] \\
\mathcal{I}_{+} & =\left[\xi_{1}, \xi_{2}\right] \cup\left[\xi_{3}, \xi_{4}\right] \cup \cdots \cup\left[\xi_{2 n-1}, \xi_{2 n}\right]
\end{aligned}
$$


For the Taylor series expansion of $G$ we have

$$
G= \begin{cases}0+g_{1}(x) y+i g_{2}(x) y^{2}+g_{3}(x) y^{3}+\ldots & x \in \mathcal{I}_{0} \\ i+g_{1}(x) y+i g_{2}(x) y^{2}+g_{3}(x) y^{3}+\ldots & x \in \mathcal{I}_{+}\end{cases}
$$

where

$$
\begin{aligned}
& g_{1}(x)=\sum_{j}(-1)^{j} \frac{1}{2} \frac{1}{\left|x-\xi_{j}\right|} \\
& g_{2}(x)=\sum_{j}(-1)^{j} \frac{1}{4} \frac{\operatorname{sign}\left(x-\xi_{j}\right)}{\left|x-\xi_{j}\right|^{2}} \\
& g_{3}(x)=\sum_{j}(-1)^{j+1} \frac{1}{4} \frac{1}{\left|x-\xi_{j}\right|^{3}}
\end{aligned}
$$

For the calculation of $b_{I}$ we also need the Taylor series expansion of $\Phi$ defined in (C.10):

$$
\Phi=L^{3}\left(\phi_{0}(x)+\phi_{2}(x) y^{2}+\phi_{4}(x) y^{4}+\ldots\right)
$$

where

$$
\begin{aligned}
& \phi_{0}(x)=\sum_{j}(-1)^{j}\left|x-\xi_{j}\right| \\
& \phi_{2}(x)=\sum_{j}(-1)^{j} \frac{1}{2} \frac{1}{\left|x-\xi_{j}\right|} \\
& \phi_{4}(x)=\sum_{j}(-1)^{j+1} \frac{1}{8} \frac{1}{\left|x-\xi_{j}\right|^{3}}
\end{aligned}
$$

Note that $g_{1}=\phi_{2}$ and $g_{3}=2 \phi_{4}$ which will be important in the expansion of the action. The combinations of metric functions appearing in (5.9) can be expanded as follows:

$$
\left(\frac{f_{2} f_{3}}{f_{1}}\right)^{3}= \begin{cases}\frac{L^{3}\left(g_{1}^{2}-g_{2}\right)^{2}}{g_{1}^{2}+g_{2}} y^{3}+O\left(y^{5}\right) & x \in \mathcal{I}_{0} \\ -\frac{L^{3}\left(g_{1}^{2}+g_{2}\right)^{2}}{2\left(g_{1}^{2}+2 g_{2}\right)} y^{3}+O\left(y^{5}\right) & x \in \mathcal{I}_{+}\end{cases}
$$

and

$$
\left(\frac{f_{1} f_{3}}{f_{2}}\right)^{3}=\left\{\begin{array}{l}
-\frac{L^{3}\left(g_{1}^{2}+g_{2}\right)^{2}}{g_{1}^{2}-g_{2}} y^{3}+O\left(y^{5}\right) x \in \mathcal{I}_{0} \\
\frac{4 L^{3}}{g_{1}^{4}+3 g_{1}^{2} g_{2}+2 g_{2}^{2}} \frac{1}{y^{3}}+O\left(\frac{1}{y}\right) x \in \mathcal{I}_{+}
\end{array}\right.
$$

and

$$
\left(\frac{f_{1} f_{2}}{f_{3}}\right)^{3}= \begin{cases}-\frac{8 L^{3}}{\left(g_{1}^{4}-g_{2}^{2}\right)} \frac{1}{y^{3}}+O\left(\frac{1}{y}\right) & x \in \mathcal{I}_{0} \\ -\frac{4 L^{3}\left(g_{1}^{2}+2 g_{2}\right)^{2}}{g_{1}^{2}+g_{2}} y^{3}+O\left(y^{5}\right) & x \in \mathcal{I}_{+}\end{cases}
$$


The expansion of $b_{I}$ with $I=1,2,3$ works the same way, but there are some cancellations due to the relations of the expansion coefficients for $G$ and $\Phi$ mentioned above. We find

$$
b_{1}=\left\{\begin{array}{l}
L^{3}\left(\frac{4 g_{1}}{g_{1}^{2}-g_{2}}-2 \phi_{0}+4 x+O\left(y^{2}\right)\right) x \in \mathcal{I}_{0} \\
L^{3}\left(\frac{4 g_{1}}{g_{1}^{2}+g_{2}}-2 \phi_{0}+4 x+O\left(y^{2}\right)\right) x \in \mathcal{I}_{+}
\end{array}\right.
$$

Since the subleading term is of order $y^{2}$ as $y \rightarrow 0$ we find that $\partial_{y}\left(b_{1}^{2}\right)$ is of order $y$. Also note that the metric factor (D.6) is of order $y^{3}$ as $y \rightarrow 0$. Thus we find that the contribution to the action coming from $b_{1}$ vanishes at $y=0$ and hence does not contribute.

The Taylor expansion of $b_{2}$ is given by

$$
b_{2}= \begin{cases}L^{3}\left(-\frac{4 g_{1}}{g_{1}^{2}+g_{2}}+2 \phi_{0}+4 x+O\left(y^{2}\right)\right) & x \in \mathcal{I}_{0} \\ L^{3}\left(\left(2 \phi_{0}+4 x\right)+\left(g_{1}^{3}+3 g_{1} g_{2}-g_{3}\right) y^{4}+O\left(y^{6}\right)\right) & x \in \mathcal{I}_{+}\end{cases}
$$

It is important to note that for $x \in \mathcal{I}_{+}$we find that $\partial_{y}\left(b_{2}\right)^{2}$ will behave as $y^{3}$ as $y \rightarrow 0$ and together with the behavior of the metric factor (D.7) produces a finite contribution to the action.

Similarly the Taylor expansion for $b_{3}$ is given by

$$
b_{3}= \begin{cases}L^{3}\left(-\phi_{0}+\left(g_{1}^{3}+\frac{g_{3}}{2}\right) y^{4}+O\left(y^{5}\right)\right) & x \in \mathcal{I}_{0} \\ L^{3}\left(-\frac{g_{1}}{g_{1}^{2}+2 g_{2}}-\phi_{0}+O\left(y^{2}\right)\right) & x \in \mathcal{I}_{+}\end{cases}
$$

In a similar manner as for $b_{2}$ we note that for $x \in \mathcal{I}_{0}$ the $\partial_{y}\left(b_{3}\right)^{2}$ term will be of order $y^{3}$ which together with the behavior of the metric factor (D.8) will produce a finite contribution to the action at $y=0$.

Summarizing we find that

$$
\begin{aligned}
& \lim _{y \rightarrow 0} \frac{f_{2}^{3} f_{3}^{2}}{2 f_{1}^{3}} \partial_{y}\left(b_{1}^{2}\right)=0, \quad x \in \mathbb{R} \\
& \lim _{y \rightarrow 0} \frac{f_{1}^{3} f_{3}^{3}}{2 f_{2}^{3}} \partial_{y}\left(b_{2}^{2}\right)= \begin{cases}0 & x \in \mathcal{I}_{0} \\
\frac{32 L^{9}\left(g_{1}^{3}+3 g_{1} g_{2}-g_{3}\right)\left(\phi_{0}+2 x\right)}{\left(g_{1}^{2}+g_{2}\right)\left(g_{1}^{2}+2 g_{2}\right)} & x \in \mathcal{I}_{+}\end{cases} \\
& \lim _{y \rightarrow 0} \frac{f_{1}^{3} f_{2}^{3}}{2 f_{3}^{3}} \partial_{y}\left(b_{3}^{2}\right)= \begin{cases}\frac{16 L^{9}\left(2 g_{1}^{3}+g_{3}\right) \phi_{0}}{\left(g_{1}^{2}-g_{2}\right)\left(g_{1}^{2}+g_{2}\right)} & x \in \mathcal{I}_{0} \\
0 & x \in \mathcal{I}_{+}\end{cases}
\end{aligned}
$$

The integration region $\mathcal{I}_{0}$ is cut off by the large $r_{c}$ cutoff and includes the intervals $\left[-r_{c}, \xi_{1}\right]$ and $\left[\xi_{2 n}, r_{c}\right]$ that are responsible for $r_{c}$ divergent terms. Using the large $|x|$ expansion one can show using the Taylor series expansions of (D.12) for large arguments that the contribution from the integral is given from the large integration limits $x_{c,+}$ and $x_{c,-}$ by

$$
\begin{aligned}
& \left.\int_{\xi_{2 n}}^{x_{c,+}} d x \frac{f_{1}^{3} f_{2}^{3}}{2 f_{3}^{3}} \partial_{y}\left(b_{3}^{2}\right)\right|_{y=0}=L^{9}\left[-16 x_{c,+}^{3}+12 m_{2} x_{c,+}^{2}+\left(16-9 m_{2}^{2}+16 m_{3}\right) x_{c,+}\right]+\text { finite } \\
& \left.\int_{x_{c,-}}^{\xi_{1}} d x \frac{f_{1}^{3} f_{2}^{3}}{2 f_{3}^{3}} \partial_{y}\left(b_{3}^{2}\right)\right|_{y=0}=L^{9}\left[-16\left|x_{c,-}\right|^{3}-12 m_{2}\left|x_{c,-}\right|^{2}+\left(16-9 m_{2}^{2}+16 m_{3}\right)\left|x_{c,-}\right|\right]+\text { finite }
\end{aligned}
$$


Using the fact that $x_{c,+}=r_{c}(0, \varepsilon)$ and $x_{c,-}=r_{c}(\pi, \varepsilon)$ together with the relation of the radial cut-off to the FG cut-off parameter $\varepsilon$ given in (2.26), one can extract the contributions of the $x$-integral that are divergent with respect to the cut-off $\varepsilon$ as follows:

$$
I_{(x)}^{\text {div }}=+\frac{L^{9}}{48 \pi G_{N}^{(11)}} \operatorname{Vol}\left(S^{3}\right)^{2} \operatorname{Vol}\left(A d S_{3}\right)\left(-\frac{256}{\varepsilon^{6}}+\frac{80-12 m_{2}^{2}+32 m_{3}}{\varepsilon^{2}}\right) .
$$

Open Access. This article is distributed under the terms of the Creative Commons Attribution License (CC-BY 4.0), which permits any use, distribution and reproduction in any medium, provided the original author(s) and source are credited.

\section{References}

[1] E. D'Hoker, J. Estes, M. Gutperle and D. Krym, Exact Half-BPS Flux Solutions in M-theory. I: Local Solutions, JHEP 08 (2008) 028 [arXiv:0806.0605] [INSPIRE].

[2] E. D'Hoker, J. Estes, M. Gutperle and D. Krym, Exact Half-BPS Flux Solutions in M-theory II: Global solutions asymptotic to $A d S_{7} \times S^{4}$, JHEP 12 (2008) 044 [arXiv:0810.4647] [INSPIRE].

[3] S. Ryu and T. Takayanagi, Holographic derivation of entanglement entropy from AdS/CFT, Phys. Rev. Lett. 96 (2006) 181602 [hep-th/0603001] [INSPIRE].

[4] S. Ryu and T. Takayanagi, Aspects of Holographic Entanglement Entropy, JHEP 08 (2006) 045 [hep-th/0605073] [INSPIRE].

[5] T. Nishioka, S. Ryu and T. Takayanagi, Holographic Entanglement Entropy: An Overview, J. Phys. A 42 (2009) 504008 [arXiv: 0905. 0932] [inSPIRE].

[6] H. Casini, M. Huerta and R.C. Myers, Towards a derivation of holographic entanglement entropy, JHEP 05 (2011) 036 [arXiv:1102.0440] [INSPIRE].

[7] S.-J. Rey and J.-T. Yee, Macroscopic strings as heavy quarks in large- $N$ gauge theory and anti-de Sitter supergravity, Eur. Phys. J. C 22 (2001) 379 [hep-th/9803001] [InSPIRE].

[8] J.M. Maldacena, Wilson loops in large-N field theories, Phys. Rev. Lett. 80 (1998) 4859 [hep-th/9803002] [INSPIRE].

[9] J. Gomis and F. Passerini, Holographic Wilson Loops, JHEP 08 (2006) 074 [hep-th/0604007] [INSPIRE].

[10] J. Gomis and F. Passerini, Wilson Loops as D3-branes, JHEP 01 (2007) 097 [hep-th/0612022] [INSPIRE].

[11] H.-C. Chang and A. Karch, Entanglement Entropy for Probe Branes, JHEP 01 (2014) 180 [arXiv:1307.5325] [INSPIRE].

[12] K. Jensen and A. O'Bannon, Holography, Entanglement Entropy and Conformal Field Theories with Boundaries or Defects, Phys. Rev. D 88 (2013) 106006 [arXiv:1309.4523] [INSPIRE].

[13] J. Estes, K. Jensen, A. O'Bannon, E. Tsatis and T. Wrase, On Holographic Defect Entropy, JHEP 05 (2014) 084 [arXiv: 1403.6475] [INSPIRE].

[14] E. D'Hoker, J. Estes and M. Gutperle, Gravity duals of half-BPS Wilson loops, JHEP 06 (2007) 063 [arXiv:0705.1004] [INSPIRE]. 
[15] O. Lunin, On gravitational description of Wilson lines, JHEP 06 (2006) 026 [hep-th/0604133] [INSPIRE].

[16] S. Yamaguchi, Bubbling geometries for half BPS Wilson lines, Int. J. Mod. Phys. A 22 (2007) 1353 [hep-th/0601089] [INSPIRE].

[17] A. Lewkowycz and J. Maldacena, Exact results for the entanglement entropy and the energy radiated by a quark, JHEP 05 (2014) 025 [arXiv: 1312.5682] [INSPIRE].

[18] O. Aharony, O. Bergman, D.L. Jafferis and J. Maldacena, $N=6$ superconformal Chern-Simons-matter theories, M2-branes and their gravity duals, JHEP 10 (2008) 091 [arXiv: 0806.1218] [INSPIRE].

[19] V. Pestun, Localization of gauge theory on a four-sphere and supersymmetric Wilson loops, Commun. Math. Phys. 313 (2012) 71 [arXiv:0712.2824] [INSPIRE].

[20] A. Kapustin, B. Willett and I. Yaakov, Exact Results for Wilson Loops in Superconformal Chern-Simons Theories with Matter, JHEP 03 (2010) 089 [arXiv:0909.4559] [InSPIRE].

[21] S.A. Gentle and M. Gutperle, Entanglement entropy of Wilson loops: Holography and matrix models, Phys. Rev. D 90 (2014) 066011 [arXiv:1407.5629] [INSPIRE].

[22] T. Okuda and D. Trancanelli, Spectral curves, emergent geometry and bubbling solutions for Wilson loops, JHEP 09 (2008) 050 [arXiv:0806.4191] [INSPIRE].

[23] J. Gomis, S. Matsuura, T. Okuda and D. Trancanelli, Wilson loop correlators at strong coupling: From matrices to bubbling geometries, JHEP 08 (2008) 068 [arXiv:0807.3330] [INSPIRE].

[24] E. Witten, Some comments on string dynamics, hep-th/9507121 [INSPIRE].

[25] A. Strominger, Open p-branes, Phys. Lett. B 383 (1996) 44 [hep-th/9512059] [INSPIRE].

[26] J.M. Maldacena, The large- $N$ limit of superconformal field theories and supergravity, Int. J. Theor. Phys. 38 (1999) 1113 [hep-th/9711200] [INSPIRE].

[27] O.J. Ganor, Six-dimensional tensionless strings in the large-N limit, Nucl. Phys. B 489 (1997) 95 [hep-th/9605201] [INSPIRE].

[28] D.E. Berenstein, R. Corrado, W. Fischler and J.M. Maldacena, The Operator product expansion for Wilson loops and surfaces in the large-N limit, Phys. Rev. D 59 (1999) 105023 [hep-th/9809188] [INSPIRE].

[29] R. Corrado, B. Florea and R. McNees, Correlation functions of operators and Wilson surfaces in the $D=6,(0,2)$ theory in the large- $N$ limit, Phys. Rev. D 60 (1999) 085011 [hep-th/9902153] [INSPIRE].

[30] B. Chen, W. He, J.-B. Wu and L. Zhang, M5-branes and Wilson Surfaces, JHEP 08 (2007) 067 [arXiv: 0707.3978] [INSPIRE].

[31] B. Chen and J.-B. Wu, Wilson-Polyakov surfaces and M-theory branes, JHEP 05 (2008) 046 [arXiv: 0802.2173] [INSPIRE].

[32] H. Mori and S. Yamaguchi, M5-branes and Wilson surfaces in AdS_7/CFT_6 correspondence, Phys. Rev. D 90 (2014) 026005 [arXiv: 1404.0930] [INSPIRE].

[33] O. Lunin, 1/2-BPS states in M-theory and defects in the dual CFTs, JHEP 10 (2007) 014 [arXiv: 0704.3442] [INSPIRE]. 
[34] D. Bak, M. Gutperle and S. Hirano, A dilatonic deformation of $A d S_{5}$ and its field theory dual, JHEP 05 (2003) 072 [hep-th/0304129] [INSPIRE].

[35] E. D'Hoker, J. Estes and M. Gutperle, Exact half-BPS Type IIB interface solutions. I. Local solution and supersymmetric Janus, JHEP 06 (2007) 021 [arXiv:0705.0022] [INSPIRE].

[36] E. D'Hoker, J. Estes, M. Gutperle, D. Krym and P. Sorba, Half-BPS supergravity solutions and superalgebras, JHEP 12 (2008) 047 [arXiv:0810.1484] [INSPIRE].

[37] C. Bachas, E. D'Hoker, J. Estes and D. Krym, M-theory Solutions Invariant under $D(2,1 ; \gamma) \oplus D(2,1 ; \gamma)$, Fortsch. Phys. 62 (2014) 207 [arXiv:1312.5477] [INSPIRE].

[38] A. Lewkowycz and J. Maldacena, Generalized gravitational entropy, JHEP 08 (2013) 090 [arXiv: 1304.4926] [INSPIRE].

[39] K. Skenderis and M. Taylor, Kaluza-Klein holography, JHEP 05 (2006) 057 [hep-th/0603016] [INSPIRE].

[40] K. Pilch, P. van Nieuwenhuizen and P.K. Townsend, Compactification of $d=11$ Supergravity on $S_{4}($ Or $11=7+4$, Too), Nucl. Phys. B 242 (1984) 377 [inSPIRE].

[41] P. van Nieuwenhuizen, The Complete Mass Spectrum of $d=11$ Supergravity Compactified on $S_{4}$ and a General Mass Formula for Arbitrary Cosets M(4), Class. Quant. Grav. 2 (1985) 1 [INSPIRE].

[42] M. Günaydin, P. van Nieuwenhuizen and N.P. Warner, General Construction of the Unitary Representations of Anti-de Sitter Superalgebras and the Spectrum of the $S^{4}$ Compactification of Eleven-dimensional Supergravity, Nucl. Phys. B 255 (1985) 63 [InSPIRE].

[43] R.G. Leigh and M. Rozali, The large- $N$ limit of the (2,0) superconformal field theory, Phys. Lett. B 431 (1998) 311 [hep-th/9803068] [INSPIRE].

[44] S. de Haro, S.N. Solodukhin and K. Skenderis, Holographic reconstruction of space-time and renormalization in the AdS/CFT correspondence, Commun. Math. Phys. 217 (2001) 595 [hep-th/0002230] [INSPIRE].

[45] P. Kraus, F. Larsen and R. Siebelink, The gravitational action in asymptotically AdS and flat space-times, Nucl. Phys. B 563 (1999) 259 [hep-th/9906127] [INSPIRE].

[46] H. Lin, O. Lunin and J.M. Maldacena, Bubbling AdS space and 1/2 BPS geometries, JHEP 10 (2004) 025 [hep-th/0409174] [INSPIRE].

[47] M.R. Douglas, On D = 5 super Yang-Mills theory and (2,0) theory, JHEP 02 (2011) 011 [arXiv: 1012.2880] [INSPIRE].

[48] J.A. Minahan, A. Nedelin and M. Zabzine, 5D super Yang-Mills theory and the correspondence to $A d S_{7} / C F T_{6}$, J. Phys. A 46 (2013) 355401 [arXiv:1304.1016] [InSPIRE].

[49] D. Young, Wilson Loops in Five-Dimensional super-Yang-Mills, JHEP 02 (2012) 052 [arXiv: 1112.3309] [INSPIRE].

[50] C.R. Graham and E. Witten, Conformal anomaly of submanifold observables in AdS/CFT correspondence, Nucl. Phys. B 546 (1999) 52 [hep-th/9901021] [InSPIRE].

[51] M. Henningson and K. Skenderis, Weyl anomaly for Wilson surfaces, JHEP 06 (1999) 012 [hep-th/9905163] [INSPIRE].

[52] A. Gustavsson, Conformal anomaly of Wilson surface observables: A Field theoretical computation, JHEP 07 (2004) 074 [hep-th/0404150] [INSPIRE]. 